Review Article

\title{
The Effects and Safety of Chinese Oral Herbal Paste on Stable Chronic Obstructive Pulmonary Disease: A Systematic Review and Meta-analysis of Randomized Controlled Trials
}

\author{
Yan Zeng, ${ }^{1,2}$ Yu Li, ${ }^{1}$ Hua Wei, ${ }^{1}$ Chan Xiong, ${ }^{1}$ Li Liao, ${ }^{1}$ Ti-wei Miao, ${ }^{2}$ Bing Mao ${ }^{D},{ }^{2}$ \\ and Juan-juan Fu $\mathbb{( 1 )}^{2}$ \\ ${ }^{1}$ Department of Pneumology, Pidu District Hospital of Traditional Chinese Medicine, \\ The Third Affiliated Hospital of Chengdu University of Traditional Chinese Medicine, Chengdu, Sichuan, China \\ ${ }^{2}$ Respiratory Group, Department of Integrated Traditional Chinese and Western Medicine, West China Hospital, \\ Sichuan University, Chengdu, China
}

Correspondence should be addressed to Juan-juan Fu; juanjuan.fu@scu.edu.cn

Received 27 September 2019; Revised 14 January 2020; Accepted 28 January 2020; Published 11 March 2020

Academic Editor: Chang G. Son

Copyright $\odot 2020$ Yan Zeng et al. This is an open access article distributed under the Creative Commons Attribution License, which permits unrestricted use, distribution, and reproduction in any medium, provided the original work is properly cited.

Background. Chinese oral herbal paste has been widely used in the treatment of chronic obstructive pulmonary disease (COPD). However, the treatment effects of herbal paste were controversial and lack evidence to support its clinical use. This study aims to systematically assess the efficacy and safety of Chinese oral herbal paste for the treatment of stable COPD. Methods. PubMed, Web of Science, CENTRAL, EMBASE, CNKI, VIP, CBM, and WANFANG database in addition to two websites of clinical trial registry were searched from respective inception to August 2019. Only randomized controlled trials (RCTs) studying Chinese herbal paste for the treatment of stable COPD were included. Methodological quality was assessed based on Cochrane risk of bias and GRADE approach. Data were analyzed using RevMan 5.3. Results. A total of 19 RCTs with 1303 individuals compared Chinese oral herbal paste and Western medicine (WM) with WM alone were included for meta-analysis. The review showed compared with WM alone, the combination of herbal paste and WM reduced exacerbation frequency. Subgroup analyses showed that after two to three months of treatment, compared with WM alone, Chinese herbal paste plus WM significantly decreased the St George's Respiratory Questionnaire (SGRQ) scores, COPD assessment test (CAT) scores, and scores of traditional Chinese medicine (TCM) syndrome, and improved clinical effective rates, lung function, and 6-minute walk distance. No serious adverse events related to herbal paste were reported. Conclusion. Current evidence showed that Chinese oral herbal paste may be an effective and welltolerated adjuvant therapy for stable COPD. Considering the risks of bias and heterogeneity, more high-quality, well-designed RCTs are still needed.

\section{Introduction}

Chronic obstructive pulmonary disease (COPD) is a leading cause of chronic morbidity and mortality worldwide due to persistent airway inflammation and airflow limitation, which induces a substantial, increasing economic and social burden $[1,2]$. A large national cross-sectional study in China has shown that the overall incidence of spirometry-defined COPD was $8.6 \%$, namely, 99.9 million people with COPD [3]. Decreased lung function, impaired activity tolerance, and chronic respiratory symptoms of patients with COPD are persistent and even aggravated due to acute exacerbations of the disease caused by various causes [4-6]. Faced with such a heavy burden, the management and prevention of COPD have important public health and health care implications [7].

The main treatments for stable COPD are inhaled longacting bronchodilators and/or glucocorticoids [2], which have been demonstrated for their beneficial effects in reducing the annual rate of exacerbations and improving health status [8-10]. However, patients still suffer from cough with sputum production, dyspnea, wheezing, and 
chest tightness due to persistent airway inflammation and airflow limitation. Additionally, repeated hospitalization due to acute exacerbations contributes to the deterioration of functional status and the related economic impact $[11,12]$. On the other hand, low inhalation flow, adherence with inhaler therapy, and inhaler misuse all can dampen the therapeutic effects of inhaling medication [13]. Therefore, seeking other effective treatments has always remaining a continuing problem for COPD.

COPD is categorized into the category of "lung distension" (feizhang in Chinese pinyin) in traditional Chinese medicine (TCM) [14]. In the theory of TCM, interior asthenia and exterior sthenia are the basic pathological property of lung distension. The pathological feature of disease is tended to interior asthenia on the stable phase of COPD, while exterior sthenia mainly corresponds to the acute exacerbation phase [14]. Lung dominates qi, which is the most essential substance constituting the body and sustaining life activities and controls breathing. Lung qi deficiency is the fundamental pathological basis of lung distension. Lung and kidney qi deficiency, qi and yin deficiency of lung and kidney, lung and spleen qi deficiency, lung, spleen, and kidney qi deficiency, or along with phlegm obstruction and blood stasis are the most common syndromes based on clinical practice and modern TCM syndrome researches [14]. Accordingly, the treatment principle of TCM mainly focuses on nourishing deficiency, and assists in removing phlegm and blood stasis for stable COPD.

Chinese herbal medicine (CHM), as one of the most popular complementary and alternative therapies, has been widely used for treatment of COPD in China and has gained increasing attention. $\mathrm{CHMs}$ are available in a variety of dosage forms, including decoction, paste, tablet, injection, granule, pill, and powder [15]. Chinese herbal paste is a thick and concentrated dosage form, which is made by adding some auxiliary materials after decocting and concentrating large compound herbal medicine $[15,16]$. Herbal paste is a kind of individualized formula prescribed based on the theory of TCM, physical constitution of patient, and underlying diseases, which is considered to be suitable for the treatment of chronic debilitated diseases exerting the effects of preventing the occurrence, progress, and recurrence of diseases as well as strengthening immunity $[17,18]$. Compared with conventional CHM decoction, Chinese herbal paste not only has advantages of higher drug concentration, bioavailability, stability, and smaller volume, but also longterm storage, convenience for carrying, and satisfying taste, which may increase patients' adherence to pharmacotherapy [19].

The efficacy and safety of Chinese oral herbal paste for COPD have been studied in many randomized controlled trials (RCTs). However, the quality of original studies and the selection of clinical outcomes produced different results. Compared with the conventional western medicine (WM) alone, some RCTs reported superior clinical outcomes of Chinese herbal paste [20-22], while the others not [23, 24]. Therefore, more comprehensive and credible evidence is needed to support the clinical application of herbal paste for stable COPD. At present, no systematic review has been performed to assess the clinical efficacy and safety of Chinese oral herbal paste in the treatment for stable COPD, which will be comprehensively evaluated in the current study.

\section{Methods}

2.1. Registration and Protocol. The systematic review and meta-analysis have been prospectively registered on international prospective register of systematic review (PROSPERO) (No. CRD42019123715). This study was conducted in accordance with the Cochrane Handbook for Systematic Reviews of Interventions [25], following our previous published study protocol including inclusion and exclusion criteria, search strategy, data collection, quality assessment, and statistical analysis of included trials [16].

2.2. Eligibility Criteria. We included only RCTs that evaluated efficacy and safety of Chinese oral herbal paste for stable COPD. Enrolled patients were diagnosed by global strategy for the diagnosis, management, and prevention of COPD [2], or guideline of Chinese Medical Association Respiratory Diseases Society for COPD [26]. Additionally, TCM syndromes needed to meet the type of deficiency syndrome, which is diagnosed according to diagnosis and treatment guideline of TCM for COPD in China [14].

The possible comparisons of intervention were as follows: (1) the combination of Chinese oral herbal paste and WM vs placebo plus same WM; (2) Chinese oral herbal paste plus WM vs same WM alone. There is no limitation on the duration, specific composition, and dosage of the herbal paste.

The exclusion criteria were as follows: (1) patients suffered from other severe respiratory illnesses; (2) studies with other TCM treatments such as Chinese medicine decoction, herbal paste acupoint application, acupuncture, and so on; and (3) the details of the trial were unclear, or the resulting data were incomplete.

The primary outcome measures were as follows: (1) acute exacerbation of COPD during follow-up after study entry; (2) quality of life measured by St George's Respiratory Questionnaire (SGRQ); and (3) symptom scores represented by COPD assessment test (CAT).

The secondary outcomes included: (1) clinical effective rates: clinical curative effect was calculated as the cumulative percentage of the syndrome score reduction (PSSR) ((total syndrome scores before treatment-total syndrome scores after treatment)/total syndrome scores before treatment $\times 100 \%$ ) based on the Guiding Principle of Clinical Research on New Drugs of TCM [27]. The scores were graded as clinical cure (PSSR $\geq 90 \%)$, marked effectivity $(70 \% \leq$ PSSR $<90 \%)$, effectivity $(30 \% \leq$ PSSR $<70 \%)$, and no-effectivity (PSSR $<30 \%)$. Clinical effective rates were defined as percentage of clinical cure, marked effectivity, and effectivity; (2) scores of TCM syndrome: namely, total scores of symptoms such as cough with sputum production, wheezing, chest tightness, fatigue, etc [27]; (3) forced expiratory volume in one second as a percentage of the predicted value ( $\mathrm{FEV}_{1} \%$ pred); (4) 6-minute walk distance (6MWD); and (5) adverse events. 
2.3. Search Strategy. We searched PubMed, Web of Science, Cochrane Central Register of Controlled Trials (CENTRAL), EMBASE, Chinese National Knowledge Infrastructure (CNKI), Chinese Scientific and Technological Periodical Database (VIP), Chinese Biomedical Database (CBM), and WANFANG Database from their respective inception to August 2019. In addition, not only the website of international clinical trial registry (http://clinicaltrials.gov/) and the website of Chinese clinical trial registry (http://www.chictr. org/), but also reference lists of articles that have been retrieved, grey literature, and conference proceedings were also searched. Searching languages included English and Chinese. Detailed search strategies and search terms or key words were shown in our published protocol [16].

2.4. Selection of Studies and Data Extraction. Two researchers (Yan Zeng and $\mathrm{Yu} \mathrm{Li)} \mathrm{initially} \mathrm{searched} \mathrm{the} \mathrm{literature}$ according to predefined search strategy, and all the retrieved literatures were imported into Endnote X9 (Thomson Reuters). After removing duplicates, the two authors (Yan Zeng and $\mathrm{Yu} \mathrm{Li}$ ) screened the title and abstract of each retrieved study for further assessment. Disputes were resolved through mutual discussion. Finally, all included trials must be reviewed by a third author (Juan-juan $\mathrm{Fu}$ ).

Two reviewers (Yan Zeng and Hua Wei) independently extracted and checked relative outcomes, and then imported data into several structured characteristic forms. The extracted data comprised characteristics of patients of included studies (e.g., age, lung function, TCM syndrome, course of disease, and the number of dropout) and study design (year of publication, sample size, TCM therapeutic principle, details of treatment and control interventions, duration of treatment and follow-up, detailed methodological information, outcome measures, and so forth). All extracted data were cross-examined by two researchers to avoid errors. Missing information was obtained by contacting the leading author of the original study, and this trial would be excluded if the relative data were still not adequately available. Any disagreement between researchers in opinion was resolved through team discussions.

2.5. Methodological Quality. The risk of bias for each included study was independently assessed by two authors (Chan Xiong and Li Liao) using the criteria described in the Cochrane systematic evaluation manual 5.1.0 [25], which included random sequence generation, allocation concealment, blinding of participants and researchers, blinding of outcome assessment, incomplete outcome data, selective reporting, and other bias. The assessment results were correspondingly classified into high risk, unclear risk, or low risk according to the above criteria. The Grading of Recommendations assessment, Development, and Evaluation (GRADE) system was used to assess the quality of evidence of each outcome measure, which included study limitations, inconsistency of results, indirectness of evidence, imprecision, and reporting bias [28]. The GRADE system divides the quality of evidence into four levels-high, moderate, low, and very low. The higher the quality of evidence, the more credible the results that the systematic review yields.

2.6. Statistical Analysis. Review Manager (RevMan) 5.3 software provided by Cochrane collaboration was used for meta-analysis. The mean difference (MD) with $95 \%$ confidence interval (CI) was used to analyze the effect size of continuous variable by inverse-variance method. In addition, dichotomous variable was presented as relative risk (RR) with corresponding 95\% CI by Mantel-Haenszel method. The $P$ value was used to test the statistical significance of each effect size, and $P$ value $<0.05$ was considered as statistically significant. Heterogeneity among included studies was evaluated statistically using the chi-square distribution and the $I^{2}$ test. All the results were represented by forest plots. We used fixed-effect model when the heterogeneity between studies was not significant $(P>0.1$ and $\left.I^{2}<50 \%\right)$. If significant heterogeneity was detected $(P \leq 0.1$ and/or $I^{2} \geq 50 \%$ ), a random-effect model was used for metaanalysis. We attempted to identify the underlying cause of heterogeneity and reduce heterogeneity through subgroup analysis. The subgroup analysis was performed by examining characteristics and differences between included studies, such as duration of intervention and follow-up, the treatment principles corresponding to TCM syndromes, severity of disease, setting, age, and so on. Sensitivity analysis of the preliminary results would be conducted to explore the robustness of the review conclusions if necessary. Publication bias would be assessed using visual funnel plot when more than ten trials were identified.

\section{Results}

3.1. Search Results. A total of 147 potential citations were initially identified. No unpublished relevant study was found. After removing duplicates and initially screening the titles and abstracts, the full text of 85 articles was reviewed. Among them, 19 studies [20-24, 29-42] with 1303 participants (663 in the herbal paste groups and 640 in the control groups) meeting the inclusion criteria were subsequently identified for analysis. Two articles reported different outcomes of the same study [33, 35], and we only included different outcome measures without duplicate calculations for the total number of patients. Details of the included studies with search process and study identification are presented (Figure 1).

3.2. Characteristics of Included Studies. All the included RCTs were conducted in China. Baseline comparisons were performed in each study, including sample size, age, and gender, which showed no statistical differences between the treatment group and control group. The included 19 studies [20-24, 29-42] all compared the combination of Chinese oral herbal paste and WM therapy with WM therapy alone. With regard to differences in diagnosis of TCM, the patients included into studies were mainly classified into five TCM syndrome types: (1) lung and kidney qi deficiency $[22,24,29,32,36,41,42]$, (2) lung and spleen qi deficiency 

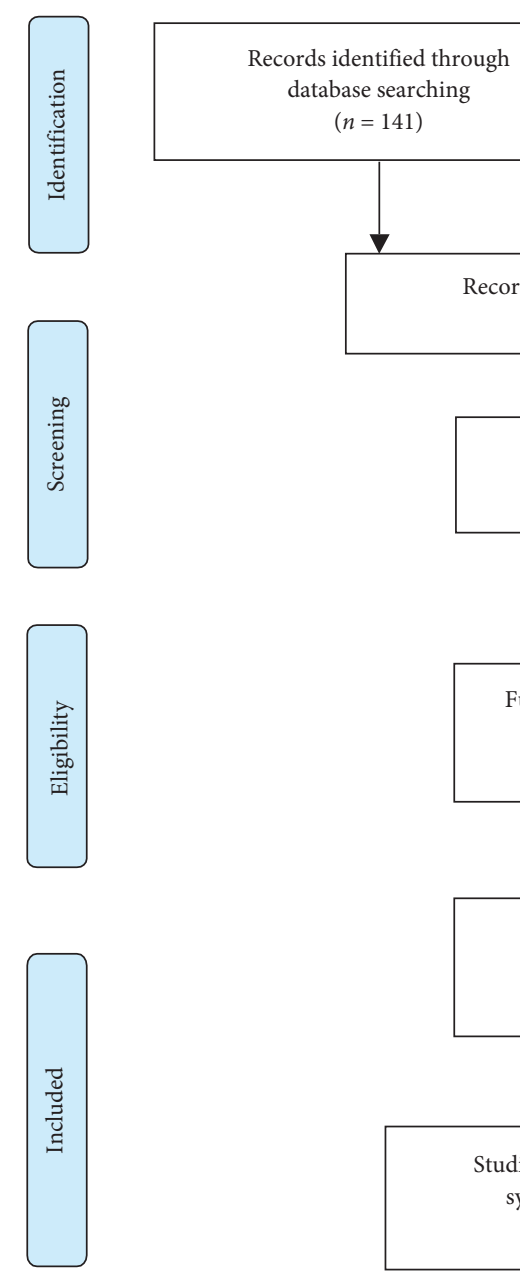
through other sources $(n=6)$

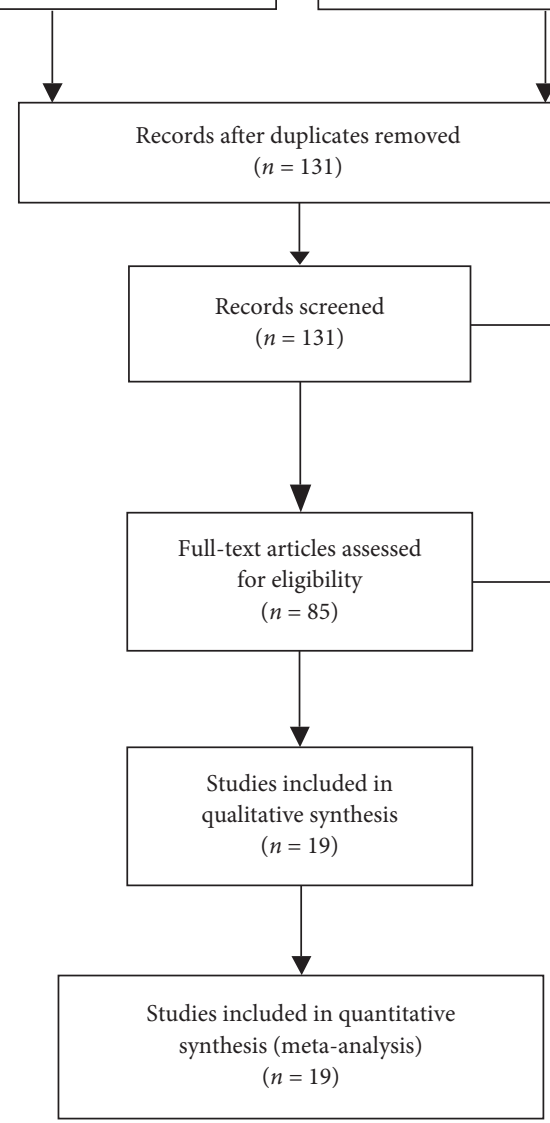

Figure 1: Study flow chart.

$[21,30,33,35,39],(3)$ lung, spleen and kidney qi deficiency $[23,31,34,38],(4)$ lung qi deficiency [20], and (5) qi and yin deficiency of lung and kidney $[37,40]$. The 19 studies all used self-modified herbal paste according to TCM syndrome type determined by symptoms and corporeity of participants, and the general principle of treatment was to strengthen lung, spleen, or kidney. In the included studies, herbal paste in different doses was given orally for different intervention durations (approximately 2-6 months), and the follow-up period varied from six months to one year. The basic characteristics of all included patients are shown in Table 1, and the details of each study design are presented in Table 2.

3.3. Quality Assessment of Included Studies. In general, most original studies showed an unclear risk of bias. All trials included were described as randomized in which 14 studies described procedures of random sequence in detail $[20,22-24,29,30,33-35,37,39-42]$. Random sequences of 11 studies were generated from the random number table $[20,22,24,29,30,33-35,39,40,42]$, and the other 3 studies only described simple randomization $[23,37,41]$. None of the studies used a placebo as a control group of herbal paste and reported whether there was allocation concealment.
Blinding of patients was not carried out in all the included studies, and blinding of researchers was performed in one trial [24]. Six studies reported withdrawals or losses to follow-up during treatment, with no significant difference in the numbers of dropouts between treatment groups and control groups (Table 1) $[22,24,29,33,35,42]$. Eight studies reported negative results $[23,24,30,33,35-37,40]$, and no protocol associated with the included studies was found. In addition, all the outcome measures specified in the Methods section of the included studies were described in the corresponding Results section. Therefore, selective reporting in the other 11 studies could not be confirmed $[20-22,29,31,32,34,38,39,41,42]$. The summary of the risk of biased items of included studies is shown in Table 3 and Figure 2.

The "GRADE profiler" of the Cochrane Collaboration Network was used to assess quality of evidence for each of the outcomes. The quality of evidence ranged from very low to moderate (Figure 3).

3.4. Outcome Measures. In the analysis of the outcome data, we tried to perform subgroup analysis through different treatment principles of Chinese herbal paste medicine, 
TABLE 1: Characteristics of the patients from the included studies.

\begin{tabular}{|c|c|c|c|c|c|c|}
\hline Author, year & $\begin{array}{c}\text { Sample } \\
\text { size }(\mathrm{T} / \mathrm{C})\end{array}$ & Age (years) $(\mathrm{T} / \mathrm{C})$ & TCM syndrome & Lung function & $\begin{array}{l}\text { Course of disease } \\
\text { (years) }(\mathrm{T} / \mathrm{C})\end{array}$ & Dropout (T/C) \\
\hline $\begin{array}{l}\text { Zhu et al. } \\
2009 \text { [24] }\end{array}$ & $37 / 37$ & $\begin{array}{c}63.72 \pm 7.52 / \\
62.91 \pm 8.34\end{array}$ & $\begin{array}{l}\text { Lung and kidney qi } \\
\text { deficiency }\end{array}$ & $\begin{array}{l}\text { Moderate to } \\
\text { severe }\end{array}$ & ND & $\begin{array}{l}\text { Lost to follow-up } \\
(n=5) / \text { lost to follow-up } \\
(n=5)\end{array}$ \\
\hline $\begin{array}{l}\text { Zhang et al. } \\
2013 \text { [29] }\end{array}$ & $53 / 54$ & $\begin{array}{c}57.38 \pm 13.21 / \\
61.16 \pm 12.93\end{array}$ & $\begin{array}{l}\text { Lung and kidney qi } \\
\text { deficiency }\end{array}$ & Mild to severe & $\begin{array}{c}11.28 \pm 7.73 / \\
10.34 \pm 5.92\end{array}$ & $\begin{array}{l}\text { Lost to follow-up } \\
(n=1) / \text { lost to follow-up } \\
(n=4)\end{array}$ \\
\hline $\begin{array}{l}\text { Wang and } \\
\text { Liu } 2013[30]\end{array}$ & $30 / 30$ & $\begin{array}{c}69.1 \pm 6.2 / \\
67.8 \pm 5.7\end{array}$ & $\begin{array}{l}\text { Lung and spleen qi } \\
\text { deficiency }\end{array}$ & $\begin{array}{l}\text { Moderate to } \\
\text { severe }\end{array}$ & ND & No/No \\
\hline $\begin{array}{l}\text { Chen et al. } \\
2014 \text { [31] }\end{array}$ & $30 / 30$ & $\begin{array}{c}58.63 \pm 10.37 / \\
57.84 \pm 11.65\end{array}$ & $\begin{array}{l}\text { Lung, spleen and kidney qi } \\
\text { deficiency }\end{array}$ & $\begin{array}{l}\text { Moderate to } \\
\text { severe }\end{array}$ & $\begin{array}{c}10.78 \pm 5.83 / \\
9.33 \pm 6.75\end{array}$ & $\mathrm{No} / \mathrm{No}$ \\
\hline $\begin{array}{l}\text { Cheng et al. } \\
2014 \text { [32] }\end{array}$ & $30 / 32$ & $54.3 / 57.5$ & $\begin{array}{l}\text { Lung and kidney qi } \\
\text { deficiency }\end{array}$ & $\begin{array}{l}\text { Moderate to } \\
\text { severe }\end{array}$ & $3 \sim 26 / 3 \sim 30$ & $\mathrm{No} / \mathrm{No}$ \\
\hline $\begin{array}{l}\text { Wang et al. } \\
2014[33]\end{array}$ & $31 / 32$ & $\begin{array}{c}70.43 \pm 9.30 / \\
67.33 \pm 9.64\end{array}$ & $\begin{array}{l}\text { Lung and spleen qi } \\
\text { deficiency }\end{array}$ & ND & ND & $\begin{array}{l}\text { Lost to follow-up } \\
(n=1) / \text { lack of } \\
\text { compliance }(n=2)\end{array}$ \\
\hline $\begin{array}{l}\text { Zhao et al. } \\
2014 \text { [34] }\end{array}$ & $45 / 45$ & $\begin{array}{c}57.1 \pm 8.5 / \\
56.3 \pm 7.3\end{array}$ & $\begin{array}{l}\text { Lung and spleen qi } \\
\text { deficiency, or lung and } \\
\text { kidney qi deficiency }\end{array}$ & $\begin{array}{l}\text { Moderate to } \\
\text { severe }\end{array}$ & $7.1 \pm 3.2 / 6.8 \pm 3.5$ & No/No \\
\hline $\begin{array}{l}\text { He et al. } 2015 \\
{[20]}\end{array}$ & $40 / 36$ & $\begin{array}{l}57.4 \pm 10.4 / \\
58.6 \pm 11.2\end{array}$ & Lung qi deficiency & ND & ND & $\mathrm{No} / \mathrm{No}$ \\
\hline $\begin{array}{l}\text { Jiang et al. } \\
2015 \text { [22] }\end{array}$ & $50 / 50$ & $65.75 / 62.09$ & $\begin{array}{l}\text { Lung and kidney qi } \\
\text { deficiency }\end{array}$ & Mild to severe & $15.23 / 13.92$ & $\begin{array}{c}\text { No/lost to follow-up } \\
(n=7)\end{array}$ \\
\hline $\begin{array}{l}\text { Wang et al. } \\
2015 \text { [35] }\end{array}$ & $31 / 32$ & $\begin{array}{c}70.43 \pm 9.30 / \\
67.33 \pm 9.64\end{array}$ & $\begin{array}{l}\text { Lung and spleen qi } \\
\text { deficiency }\end{array}$ & ND & ND & $\begin{array}{l}\text { Lost to follow-up } \\
\quad(n=1) / \text { lack of } \\
\text { compliance }(n=2)\end{array}$ \\
\hline $\begin{array}{l}\text { Wu et al. } \\
2015[36]\end{array}$ & $35 / 25$ & $\begin{array}{c}71.23 \pm 6.64 / \\
73.39 \pm 4.61\end{array}$ & $\begin{array}{l}\text { Lung and kidney qi } \\
\text { deficiency }\end{array}$ & $\mathrm{ND}$ & ND & No/No \\
\hline $\begin{array}{l}\text { Mao } 2016 \\
{[37]}\end{array}$ & $30 / 30$ & $\begin{array}{c}62.2 \pm 7.11 / \\
64.1 \pm 8.61\end{array}$ & $\begin{array}{l}\text { Qi and Yin deficiency of } \\
\text { lung and kidney }\end{array}$ & Mild to severe & $5.3 \pm 1.1 / 5.4 \pm 1.5$ & No/No \\
\hline $\begin{array}{l}\text { Zhang et al. } \\
2017 \text { [38] }\end{array}$ & $60 / 60$ & $\begin{array}{l}67.72 \pm 10.16 / \\
65.35 \pm 10.62\end{array}$ & $\begin{array}{l}\text { Lung, spleen, and kidney qi } \\
\text { deficiency }\end{array}$ & $\begin{array}{l}\text { Moderate to } \\
\text { very severe }\end{array}$ & ND & $\mathrm{No} / \mathrm{No}$ \\
\hline $\begin{array}{l}\text { Zhao and Li } \\
2017[21]\end{array}$ & $39 / 39$ & $\begin{array}{c}55.09 \pm 7.88 / \\
55.31 \pm 7.65\end{array}$ & $\begin{array}{c}\text { Lung and spleen qi } \\
\text { deficiency }\end{array}$ & Mild to severe & $\begin{array}{l}3.96 \pm 0.52 / \\
3.58 \pm 0.44\end{array}$ & $\mathrm{No} / \mathrm{No}$ \\
\hline $\begin{array}{l}\text { Deng et al. } \\
2017 \text { [39] }\end{array}$ & $41 / 39$ & $\begin{array}{c}70.04 \pm 5.12 / \\
70.59 \pm 4.83\end{array}$ & $\begin{array}{l}\text { Lung and spleen qi } \\
\text { deficiency }\end{array}$ & Mild to severe & $\begin{array}{l}8.23 \pm 3.54 / \\
8.32 \pm 3.02\end{array}$ & $\mathrm{No} / \mathrm{No}$ \\
\hline Luo 2017 [40] & $30 / 30$ & $\begin{array}{l}65.3 \pm 9.8 / \\
64.9 \pm 10.2\end{array}$ & $\begin{array}{l}\text { Qi and yin deficiency of } \\
\text { lung and kidney }\end{array}$ & Severe & $\begin{array}{c}12.8 \pm 4.7 / \\
11.3 \pm 5.1\end{array}$ & $\mathrm{No} / \mathrm{No}$ \\
\hline $\begin{array}{l}\text { Rong and } \\
\text { Ding } 2017 \\
{[41]}\end{array}$ & $30 / 30$ & $\begin{array}{c}56.5 \pm 6.2 / \\
56.1 \pm 6.3\end{array}$ & $\begin{array}{l}\text { Lung and kidney qi } \\
\text { deficiency }\end{array}$ & $\begin{array}{l}\text { Severe to very } \\
\text { severe }\end{array}$ & ND & $\mathrm{No} / \mathrm{No}$ \\
\hline $\begin{array}{l}\text { Zhang } 2018 \\
{[42]}\end{array}$ & $30 / 30$ & $\begin{array}{l}68.7 \pm 8.33 / \\
68.84 \pm 9.14\end{array}$ & $\begin{array}{l}\text { Lung and kidney qi } \\
\text { deficiency }\end{array}$ & $\begin{array}{l}\text { Mild to very } \\
\text { severe }\end{array}$ & $\begin{array}{c}10.03 \pm 4.88 / \\
10.31 \pm 4.01\end{array}$ & $\begin{array}{l}\text { Lost to follow-up } \\
(n=1) / \text { lost to follow-up } \\
(n=1)\end{array}$ \\
\hline $\begin{array}{l}\text { Xia and Sun } \\
2019[23]\end{array}$ & $30 / 30$ & $\begin{array}{l}66.93 \pm 9.11 / \\
67.23 \pm 8.92\end{array}$ & $\begin{array}{l}\text { Lung, spleen, and kidney qi } \\
\text { deficiency }\end{array}$ & $\begin{array}{l}\text { Moderate to } \\
\text { very severe }\end{array}$ & $\begin{array}{c}11.03 \pm 6.60 / \\
11.27 \pm 6.63\end{array}$ & $\mathrm{No} / \mathrm{No}$ \\
\hline
\end{tabular}

T: treatment; C: control; ND: not documented.

duration of treatment, follow-up time, disease severity etc. among studies. However, the results suggested that there was no significant change in the heterogeneity of the metaanalysis after the subgroup analysis using other factors except for the treatment and follow-up period. Therefore, we mainly conducted a meta-subgroup analysis based on differences in treatment and follow-up time.

3.4.1. Acute Exacerbations. Ten RCTs [20, 22, 23, 29, 31, $33,36,37,41,42]$ reported acute exacerbations of COPD. Seven trials $[20,23,29,31,36,37,42]$ reported exacerbation frequency. A random-effect model was used for metaanalysis. The results of the subgroup analysis showed that compared with WM alone, the combination of Chinese herbal paste and WM reduced exacerbation frequency in one year (MD: $-1.01,95 \% \mathrm{CI}:-1.61$ to $-0.41 ; P=0.001 ; I^{2}=76 \%$ ) $[23,29,31,37]$, six months (MD: $-0.71,95 \% \mathrm{CI}:-1.23$ to $\left.-0.20 ; P=0.007 ; I^{2}=82 \%\right)[36,42]$, or three months [20] (MD: $-0.91,95 \% \mathrm{CI}:-1.10$ to $-0.72 ; P<0.00001$ ) (Figure 4 ). Three RCTs $[22,33,41]$ reported separately the number of patients with one, two, or even more than two exacerbations between treatment and control groups during the study period, which could not analyzed statistically. 
TABLE 2: Study design and outcome measures of the included RCTs.

\begin{tabular}{|c|c|c|c|c|c|}
\hline \multirow{2}{*}{$\begin{array}{l}\text { Author, } \\
\text { Year }\end{array}$} & \multirow{2}{*}{$\begin{array}{l}\text { TCM therapeutic } \\
\text { principle }\end{array}$} & \multicolumn{2}{|c|}{ Interventional measures } & \multirow{2}{*}{$\begin{array}{l}\text { Duration/ } \\
\text { follow-up }\end{array}$} & \multirow{2}{*}{ Outcomes } \\
\hline & & Trial group & Control group & & \\
\hline $\begin{array}{l}\text { Zhu et al. } \\
2009 \text { [24] }\end{array}$ & $\begin{array}{l}\text { Nourishing the } \\
\text { kidney and lung }\end{array}$ & $\begin{array}{l}6 \mathrm{~g} \text { of Bufei Yishen paste, three times } \\
\text { daily; two spray of ipratropium } \\
\text { bromide, three times daily; } 30 \mathrm{mg} \text { of } \\
\text { ambroxol, three times daily }\end{array}$ & $\begin{array}{c}\text { Two spray of ipratropium } \\
\text { bromide, three times daily; } \\
30 \mathrm{mg} \text { of ambroxol, three } \\
\text { times daily }\end{array}$ & $\begin{array}{l}\text { Three } \\
\text { months/no }\end{array}$ & (1) (2) (3) (7) (8) \\
\hline $\begin{array}{l}\text { Zhang et al. } \\
2013 \text { [29] }\end{array}$ & $\begin{array}{l}\text { Nourishing the } \\
\text { kidney and lung }\end{array}$ & $\begin{array}{l}25 \mathrm{ml} \text { of herbal paste, twice daily; } \\
\text { conventional Western medicine }\end{array}$ & $\begin{array}{c}\text { Conventional Western } \\
\text { medicine }\end{array}$ & $\begin{array}{l}\text { Two months/ } \\
\text { one year }\end{array}$ & (1) (2) (5) (7) \\
\hline $\begin{array}{l}\text { Wang and } \\
\text { Liu } 2013 \\
{[30]}\end{array}$ & $\begin{array}{l}\text { Nourishing the } \\
\text { spleen and lung }\end{array}$ & $\begin{array}{l}5 \mathrm{~g} \text { of Feikang paste, once daily, and } \\
\text { after a week, } 5 \mathrm{~g} \text { of Feikang paste, twice } \\
\text { daily; one puff of seretide, twice daily; } \\
0.2 \mathrm{~g} \text { of theophylline sustained release } \\
\text { tablets, twice daily }\end{array}$ & $\begin{array}{l}\text { One puff of seretide, twice } \\
\text { daily; } 0.2 \mathrm{~g} \text { of theophylline } \\
\text { sustained release tablets, twice } \\
\text { daily }\end{array}$ & $\begin{array}{l}\text { Two months/ } \\
\text { no }\end{array}$ & (1) (2) (4) (6) (7) (8) \\
\hline $\begin{array}{l}\text { Chen et al. } \\
2014[31]\end{array}$ & $\begin{array}{l}\text { Nourishing the } \\
\text { lung, spleen and } \\
\text { kidney }\end{array}$ & $\begin{array}{l}20 \mathrm{~g} \text { of Shuyuhuo paste, twice daily; } \\
\text { one puff of tiotropium, once daily }\end{array}$ & $\begin{array}{l}\text { One puff of tiotropium, once } \\
\text { daily }\end{array}$ & $\begin{array}{l}\text { Three } \\
\text { months/one } \\
\text { year }\end{array}$ & (2) (5) \\
\hline $\begin{array}{l}\text { Cheng et al. } \\
2014 \text { [32] }\end{array}$ & $\begin{array}{l}\text { Nourishing the } \\
\text { kidney and lung }\end{array}$ & $\begin{array}{l}10 \mathrm{ml} \text { of Bushen Gujin paste, twice } \\
\text { daily; conventional Western medicine }\end{array}$ & $\begin{array}{l}\text { Conventional Western } \\
\text { medicine }\end{array}$ & $\begin{array}{l}\text { Three } \\
\text { months/no }\end{array}$ & (1) (4) (7) \\
\hline $\begin{array}{l}\text { Wang et al. } \\
2014[33]\end{array}$ & $\begin{array}{l}\text { Nourishing the } \\
\text { spleen and lung }\end{array}$ & $\begin{array}{l}10 \mathrm{~g} \text { of Baibu Yangfei paste, three } \\
\text { times daily; one puff of tiotropium, } \\
\text { once daily }\end{array}$ & $\begin{array}{l}\text { One puff of tiotropium, once } \\
\text { daily }\end{array}$ & $\begin{array}{l}\text { Six months/ } \\
\text { no }\end{array}$ & (2)(5) (7) \\
\hline $\begin{array}{l}\text { Zhao et al. } \\
2014 \text { [34] }\end{array}$ & $\begin{array}{l}\text { Nourishing the } \\
\text { lung, spleen and } \\
\text { kidney }\end{array}$ & $\begin{array}{l}15 \mathrm{~g} \text { of Bufei Huazhuo paste, three } \\
\text { times daily; one puff of seretide, twice } \\
\text { daily }\end{array}$ & $\begin{array}{c}\text { One puff of seretide, twice } \\
\text { daily }\end{array}$ & $\begin{array}{l}\text { Six months/ } \\
\text { no }\end{array}$ & (2) (3) (7) \\
\hline $\begin{array}{l}\text { He et al. } \\
2015[20]\end{array}$ & $\begin{array}{l}\text { Nourishing the } \\
\text { lung }\end{array}$ & $\begin{array}{l}20 \mathrm{ml} \text { of dongling paste, once daily; } \\
\text { one puff of seretide, twice daily; one } \\
\text { puff of tiotropium, once daily. }\end{array}$ & $\begin{array}{c}\text { One puff of seretide, twice } \\
\text { daily; one puff of tiotropium, } \\
\text { once daily. }\end{array}$ & $\begin{array}{l}\text { Three } \\
\text { months/no }\end{array}$ & (3) (4) (5) \\
\hline $\begin{array}{l}\text { Jiang et al. } \\
2015 \text { [22] }\end{array}$ & $\begin{array}{l}\text { Nourishing the } \\
\text { kidney and lung }\end{array}$ & $\begin{array}{l}15 \mathrm{~g} \text { of Yishen Lianfei paste, twice } \\
\text { daily; conventional Western medicine. }\end{array}$ & $\begin{array}{l}\text { Conventional Western } \\
\text { medicine }\end{array}$ & $\begin{array}{l}\text { Three } \\
\text { months/six } \\
\text { months }\end{array}$ & (1)(5) (8) \\
\hline $\begin{array}{l}\text { Wang et al. } \\
2015 \text { [35] }\end{array}$ & $\begin{array}{l}\text { Nourishing the } \\
\text { spleen and lung }\end{array}$ & $\begin{array}{l}10 \mathrm{~g} \text { of Baibu Yangfei paste, three } \\
\text { times daily; one puff of tiotropium, } \\
\text { once daily }\end{array}$ & $\begin{array}{c}\text { One puff of tiotropium, once } \\
\text { daily }\end{array}$ & $\begin{array}{l}\text { Six months/ } \\
\text { no }\end{array}$ & (1) (4) \\
\hline $\begin{array}{l}\text { Wu et al. } \\
2015[36]\end{array}$ & $\begin{array}{l}\text { Nourishing the } \\
\text { kidney and lung }\end{array}$ & $\begin{array}{l}15 \mathrm{~g} \text { of Yifei paste, twice daily; one puff } \\
\text { of tiotropium, once daily }\end{array}$ & $\begin{array}{l}\text { One puff of tiotropium, once } \\
\text { daily }\end{array}$ & $\begin{array}{l}\text { Three } \\
\text { months/six } \\
\text { months }\end{array}$ & (1) (4) (5) (6) \\
\hline $\begin{array}{l}\text { Mao } 2016 \\
{[37]}\end{array}$ & $\begin{array}{l}\text { Nourishing the } \\
\text { kidney and lung }\end{array}$ & $\begin{array}{l}15 \mathrm{ml} \text { of Pingchuan Guben paste, } \\
\text { twice daily; 200ug of Budesonide } \\
\text { aerosol, two times daily; } 100 \sim 200 \text { ug of } \\
\text { ventolin, } 8 \sim 12 \text { times daily }\end{array}$ & $\begin{array}{l}\text { 200ug of Budesonide aerosol, } \\
\text { twice daily; 100 200ug of } \\
\text { ventolin, 8 12 times daily }\end{array}$ & $\begin{array}{l}\text { Three } \\
\text { months/one } \\
\text { year }\end{array}$ & (1) (2) (5) (6) (7) \\
\hline $\begin{array}{l}\text { Zhang et al. } \\
2017 \text { [38] }\end{array}$ & $\begin{array}{l}\text { Nourishing the } \\
\text { lung, spleen and } \\
\text { kidney }\end{array}$ & $\begin{array}{l}20 \mathrm{~g} \text { of Bufei Zhike paste, twice daily; } \\
\text { one puff of seretide, twice daily; }\end{array}$ & $\begin{array}{l}\text { One puff of seretide, twice } \\
\text { daily; }\end{array}$ & $\begin{array}{l}\text { Six months/ } \\
\text { no }\end{array}$ & (2) \\
\hline $\begin{array}{l}\text { Zhao and Li } \\
2017 \text { [21] }\end{array}$ & $\begin{array}{l}\text { Nourishing the } \\
\text { spleen and lung }\end{array}$ & $\begin{array}{l}\text { One spoon of Bufei Huatan paste, } \\
\text { once or twice daily; one puff of } \\
\text { seretide, twice daily }\end{array}$ & $\begin{array}{l}\text { One puff of seretide, twice } \\
\text { daily }\end{array}$ & $\begin{array}{l}\text { Three } \\
\text { months/no }\end{array}$ & (1) (2) (3) \\
\hline $\begin{array}{l}\text { Deng et al. } \\
2017 \text { [39] }\end{array}$ & $\begin{array}{l}\text { Nourishing the } \\
\text { spleen and lung }\end{array}$ & $\begin{array}{l}10 \mathrm{ml} \text { of Jianpi Lifei paste, twice daily; } \\
\text { conventional Western medicine }\end{array}$ & $\begin{array}{l}\text { Conventional Western } \\
\text { medicine }\end{array}$ & $\begin{array}{l}\text { Three } \\
\text { months/six } \\
\text { months }\end{array}$ & (4) \\
\hline $\begin{array}{l}\text { Luo } 2017 \\
{[40]}\end{array}$ & $\begin{array}{l}\text { Nourishing the } \\
\text { kidney and lung }\end{array}$ & $\begin{array}{l}20 \mathrm{~g} \text { of Fufei Gushen paste, twice daily; } \\
\text { one puff of seretide, twice daily }\end{array}$ & $\begin{array}{c}\text { One puff of seretide, twice } \\
\text { daily }\end{array}$ & $\begin{array}{l}\text { Three } \\
\text { months/no }\end{array}$ & (1) (6) (8) \\
\hline $\begin{array}{l}\text { Rong and } \\
\text { Ding } 2017 \\
{[41]}\end{array}$ & $\begin{array}{l}\text { Nourishing the } \\
\text { kidney and lung }\end{array}$ & $\begin{array}{l}20 \mathrm{ml} \text { of Zijin Bushui paste, once daily, } \\
\text { and after a week, } 20 \mathrm{ml} \text { of Zijin Bushui } \\
\text { paste, twice daily; one puff of seretide, } \\
\text { twice daily; } 0.1 \mathrm{~g} \text { of theophylline } \\
\text { sustained release tablets, twice daily }\end{array}$ & $\begin{array}{l}\text { One puff of seretide, twice } \\
\text { daily; } 0.1 \mathrm{~g} \text { of theophylline } \\
\text { sustained release tablets, twice } \\
\text { daily }\end{array}$ & $\begin{array}{l}\text { Two months/ } \\
\text { no }\end{array}$ & (2) (3) (4) (5) (8) \\
\hline $\begin{array}{l}\text { Zhang } 2018 \\
{[42]}\end{array}$ & $\begin{array}{l}\text { Nourishing the } \\
\text { kidney and lung }\end{array}$ & $\begin{array}{l}25 \mathrm{~g} \text { of Bufei Yishen paste, twice daily; } \\
\text { conventional Western medicine }\end{array}$ & $\begin{array}{l}\text { Conventional Western } \\
\text { medicine }\end{array}$ & $\begin{array}{l}\text { Two months/ } \\
\text { six months }\end{array}$ & (1) (2) (4) (5) (6) (7) (8) \\
\hline $\begin{array}{l}\text { Xia and Sun } \\
2019[23]\end{array}$ & $\begin{array}{l}\text { Nourishing the } \\
\text { lung, spleen and } \\
\text { kidney }\end{array}$ & $\begin{array}{l}\text { One spoon of Gushen Bupi paste, } \\
\text { twice daily; one puff of seretide, twice } \\
\text { daily }\end{array}$ & $\begin{array}{l}\text { One puff of seretide, twice } \\
\text { daily }\end{array}$ & $\begin{array}{l}\text { Three } \\
\text { months/nine } \\
\text { months }\end{array}$ & (1)(4)(5) \\
\hline
\end{tabular}


TABLE 3: Assessment of risk of bias for included RCTs.

\begin{tabular}{|c|c|c|c|c|c|c|c|}
\hline Author, year & $\begin{array}{c}\text { Random } \\
\text { sequence } \\
\text { generation }\end{array}$ & $\begin{array}{l}\text { Allocation } \\
\text { concealment }\end{array}$ & $\begin{array}{l}\text { Blinding of } \\
\text { participants and } \\
\text { researchers }\end{array}$ & $\begin{array}{l}\text { Blinding of } \\
\text { outcome } \\
\text { assessment }\end{array}$ & $\begin{array}{l}\text { Incomplete } \\
\text { outcome data }\end{array}$ & $\begin{array}{l}\text { Selective } \\
\text { reporting }\end{array}$ & $\begin{array}{l}\text { Other } \\
\text { bias }\end{array}$ \\
\hline $\begin{array}{l}\text { Zhu et al. } \\
2009 \text { [24] }\end{array}$ & Low risk & Unclear risk & High risk & Unclear risk & Low risk & Low risk & $\begin{array}{c}\text { Unclear } \\
\text { risk }\end{array}$ \\
\hline $\begin{array}{l}\text { Zhang et al. } \\
2013 \text { [29] }\end{array}$ & Low risk & Unclear risk & High risk & Unclear risk & Low risk & Unclear risk & $\begin{array}{c}\text { Unclear } \\
\text { risk }\end{array}$ \\
\hline $\begin{array}{l}\text { Wang and Liu } \\
2013 \text { [30] }\end{array}$ & Low risk & Unclear risk & High risk & Unclear risk & Low risk & Low risk & $\begin{array}{c}\text { Unclear } \\
\text { risk }\end{array}$ \\
\hline $\begin{array}{l}\text { Chen et al. } \\
2014 \text { [31] }\end{array}$ & Unclear risk & Unclear risk & High risk & Unclear risk & Low risk & Unclear risk & $\begin{array}{c}\text { Unclear } \\
\text { risk }\end{array}$ \\
\hline $\begin{array}{l}\text { Cheng et al. } \\
2014 \text { [32] }\end{array}$ & Unclear risk & Unclear risk & High risk & Unclear risk & Low risk & Unclear risk & $\begin{array}{c}\text { Unclear } \\
\text { risk }\end{array}$ \\
\hline $\begin{array}{l}\text { Wang et al. } \\
2014 \text { [33] }\end{array}$ & Low risk & Unclear risk & High risk & Unclear risk & Low risk & Low risk & $\begin{array}{c}\text { Unclear } \\
\text { risk }\end{array}$ \\
\hline $\begin{array}{l}\text { Zhao et al. } \\
2014 \text { [34] }\end{array}$ & Low risk & Unclear risk & High risk & Unclear risk & Low risk & Unclear risk & $\begin{array}{c}\text { Unclear } \\
\text { risk }\end{array}$ \\
\hline $\begin{array}{l}\text { He et al. } 2015 \\
{[20]}\end{array}$ & Low risk & Unclear risk & High risk & Unclear risk & Low risk & Unclear risk & $\begin{array}{c}\text { Unclear } \\
\text { risk }\end{array}$ \\
\hline $\begin{array}{l}\text { Jiang et al. } \\
2015 \text { [22] }\end{array}$ & Low risk & Unclear risk & High risk & Unclear risk & Low risk & Unclear risk & $\begin{array}{c}\text { Unclear } \\
\text { risk }\end{array}$ \\
\hline $\begin{array}{l}\text { Wang et al. } \\
2015 \text { [35] }\end{array}$ & Low risk & Unclear risk & High risk & Unclear risk & Low risk & Low risk & $\begin{array}{c}\text { Unclear } \\
\text { risk }\end{array}$ \\
\hline $\begin{array}{l}\text { Wu et al. } 2015 \\
\text { [36] }\end{array}$ & Unclear risk & Unclear risk & High risk & Unclear risk & Low risk & Low risk & $\begin{array}{c}\text { Unclear } \\
\text { risk }\end{array}$ \\
\hline $\begin{array}{l}\text { Mao } 2016 \\
{[37]}\end{array}$ & Low risk & Unclear risk & High risk & Unclear risk & Low risk & Low risk & $\begin{array}{c}\text { Unclear } \\
\text { risk }\end{array}$ \\
\hline $\begin{array}{l}\text { Zhang et al. } \\
2017 \text { [38] }\end{array}$ & Unclear risk & Unclear risk & High risk & Unclear risk & Low risk & Unclear risk & $\begin{array}{c}\text { Unclear } \\
\text { risk }\end{array}$ \\
\hline $\begin{array}{l}\text { Zhao and Li } \\
2017[21]\end{array}$ & Unclear risk & Unclear risk & High risk & Unclear risk & Low risk & Unclear risk & $\begin{array}{c}\text { Unclear } \\
\text { risk }\end{array}$ \\
\hline $\begin{array}{l}\text { Deng et al. } \\
2017 \text { [39] }\end{array}$ & Low risk & Unclear risk & High risk & Unclear risk & Low risk & Unclear risk & $\begin{array}{c}\text { Unclear } \\
\text { risk }\end{array}$ \\
\hline Luo 2017 [40] & Low risk & Unclear risk & High risk & Unclear risk & Low risk & Low risk & $\begin{array}{c}\text { Unclear } \\
\text { risk }\end{array}$ \\
\hline $\begin{array}{l}\text { Rong and } \\
\text { Ding } 2017 \\
{[41]}\end{array}$ & Low risk & Unclear risk & High risk & Unclear risk & Low risk & Unclear risk & $\begin{array}{l}\text { Unclear } \\
\text { risk }\end{array}$ \\
\hline $\begin{array}{l}\text { Zhang } 2018 \\
\text { [42] }\end{array}$ & Low risk & Unclear risk & High risk & Unclear risk & Low risk & Unclear risk & $\begin{array}{c}\text { Unclear } \\
\text { risk }\end{array}$ \\
\hline $\begin{array}{l}\text { Xia and Sun } \\
2019[23]\end{array}$ & Low risk & Unclear risk & High risk & Unclear risk & Low risk & Low risk & $\begin{array}{c}\text { Unclear } \\
\text { risk }\end{array}$ \\
\hline
\end{tabular}

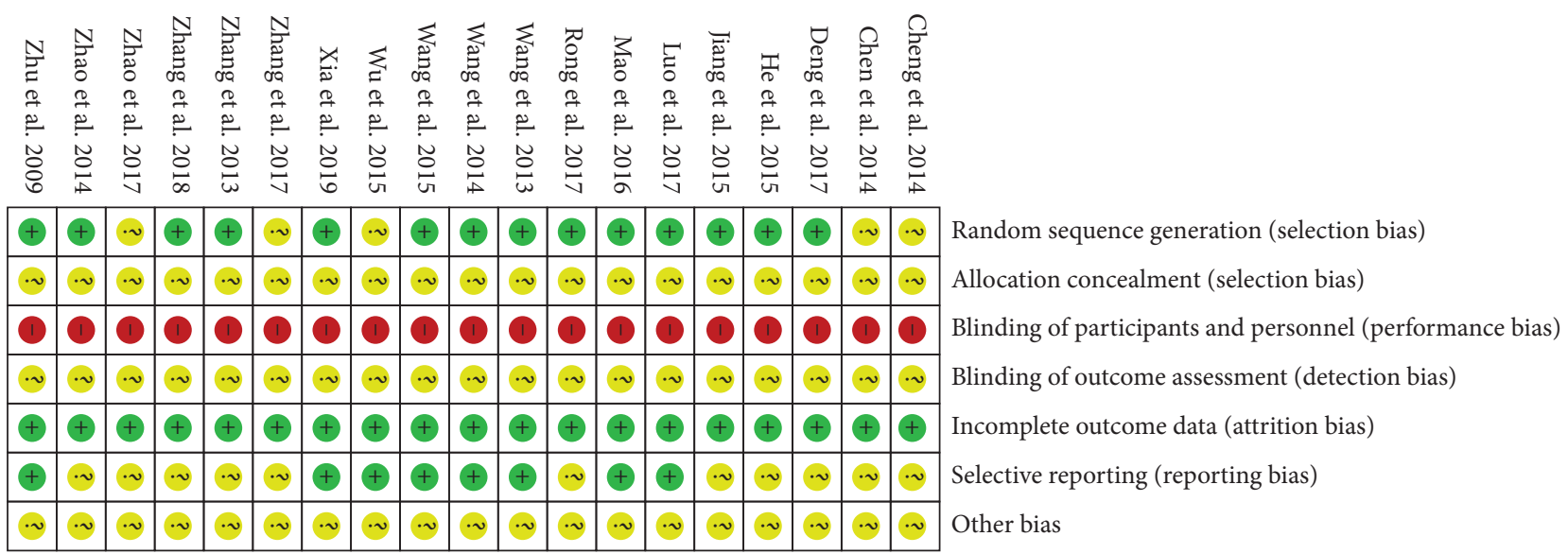

FIgURE 2: Risk of bias summary. 


\begin{tabular}{|c|c|c|c|c|c|c|c|c|c|c|c|c|}
\hline \multirow[b]{2}{*}{$\begin{array}{l}\text { No of } \\
\text { studies }\end{array}$} & \multirow[b]{2}{*}{ Design } & \multirow[b]{2}{*}{$\begin{array}{l}\text { Risk of } \\
\text { bias }\end{array}$} & \multicolumn{2}{|c|}{ Quality assessment } & \multirow[b]{2}{*}{ Imprecision } & \multirow[b]{2}{*}{$\begin{array}{c}\text { Other } \\
\text { considerations }\end{array}$} & \multicolumn{2}{|l|}{ No of patients } & \multicolumn{2}{|c|}{ Effect } & \multirow[b]{2}{*}{ Quality } & \multirow[b]{2}{*}{ Importance } \\
\hline & & & Inconsistency & Indirectness & & & $\begin{array}{c}\text { Chinese herbal paste }+ \\
\text { WM }\end{array}$ & + & $\begin{array}{l}\text { Relative } \\
\text { (95\% CI) }\end{array}$ & Absolute & & \\
\hline \multicolumn{13}{|c|}{ Frequency of acute exacerbation } \\
\hline 7 & $\begin{array}{c}\text { Randomized } \\
\text { trials }\end{array}$ & Serious $^{1}$ & $\begin{array}{l}\text { No serious } \\
\text { inconsistency }\end{array}$ & $\begin{array}{l}\text { No serious } \\
\text { indirectness }\end{array}$ & $\begin{array}{l}\text { No serious } \\
\text { imprecision }\end{array}$ & None & 246 & 230 & - & $\begin{array}{c}\text { not } \\
\text { pooled }\end{array}$ & $\begin{array}{c}\oplus \oplus \oplus \mathrm{O} \\
\text { Moderate }\end{array}$ & Critial \\
\hline \multicolumn{13}{|c|}{ SGRQ scores } \\
\hline 5 & $\begin{array}{l}\text { Randomized } \\
\text { trials }\end{array}$ & Serious ${ }^{1}$ & $\begin{array}{l}\text { No serious } \\
\text { inconsistency }\end{array}$ & $\begin{array}{l}\text { No serious } \\
\text { indirectness }\end{array}$ & $\begin{array}{l}\text { No serious } \\
\text { imprecision }\end{array}$ & None & 186 & 182 & - & $\begin{array}{c}\text { not } \\
\text { pooled }\end{array}$ & $\begin{array}{l}\oplus \oplus \oplus \mathrm{O} \\
\text { Moderate }\end{array}$ & Critial \\
\hline \multicolumn{13}{|c|}{ CAT scores } \\
\hline 9 & $\begin{array}{c}\text { Randomized } \\
\text { trials }\end{array}$ & Serious ${ }^{1}$ & $\begin{array}{l}\text { No serious } \\
\text { inconsistency }\end{array}$ & $\begin{array}{l}\text { No serious } \\
\text { indirectness }\end{array}$ & $\begin{array}{l}\text { No serious } \\
\text { imprecision }\end{array}$ & None & 295 & 281 & - & $\begin{array}{c}\text { not } \\
\text { pooled }\end{array}$ & $\begin{array}{c}\oplus \oplus \oplus \mathrm{O} \\
\text { Moderate }\end{array}$ & Critial \\
\hline \multicolumn{13}{|c|}{ Clinical effective rates } \\
\hline $\begin{array}{l}11 \\
\text { Scores o }\end{array}$ & $\begin{array}{c}\text { Rrandomized } \\
\text { trials } \\
\text { CM syndrome }\end{array}$ & Serious $^{1}$ & $\begin{array}{l}\text { No serious } \\
\text { inconsistency }\end{array}$ & $\begin{array}{l}\text { No serious } \\
\text { indirectness }\end{array}$ & $\begin{array}{l}\text { No serious } \\
\text { imprecision }\end{array}$ & Neporting bias ${ }^{2}$ & $\begin{array}{r}372 / 407 \\
(91.4 \%)\end{array}$ & $\begin{array}{r}297 / 405 \\
(73.3 \%)\end{array}$ & $\begin{array}{c}\text { Not } \\
\text { pooled }\end{array}$ & $\begin{array}{c}\text { not } \\
\text { pooled }\end{array}$ & $\underset{\text { Low }}{\oplus \oplus \mathrm{OO}}$ & Important \\
\hline 8 & $\begin{array}{l}\text { Randomized } \\
\text { trials }\end{array}$ & Serious $^{1}$ & $\begin{array}{l}\text { No serious } \\
\text { inconsistency }\end{array}$ & $\begin{array}{l}\text { No serious } \\
\text { indirectness }\end{array}$ & $\begin{array}{l}\text { No serious } \\
\text { imprecision }\end{array}$ & None & 278 & 278 & - & $\begin{array}{c}\text { not } \\
\text { pooled }\end{array}$ & $\begin{array}{l}\oplus \oplus \oplus \mathrm{O} \\
\text { Moderate }\end{array}$ & Important \\
\hline \multicolumn{13}{|c|}{ FEV1\%pre } \\
\hline 12 & $\begin{array}{l}\text { Randomized } \\
\text { trials }\end{array}$ & Serious $^{1}$ & $\begin{array}{l}\text { No serious } \\
\text { inconsistency }\end{array}$ & $\begin{array}{l}\text { No serious } \\
\text { indirectness }\end{array}$ & $\begin{array}{l}\text { No serious } \\
\text { imprecision }\end{array}$ & Neporting bias ${ }^{2}$ & 417 & 400 & - & $\begin{array}{c}\text { not } \\
\text { pooled }\end{array}$ & $\begin{array}{l}\oplus \mathrm{OOO} \\
\text { Very low }\end{array}$ & Important \\
\hline 6MWD & & & & & & & & & & & & \\
\hline 5 & $\begin{array}{c}\text { Randomized } \\
\text { trials }\end{array}$ & Serious $^{1}$ & $\begin{array}{l}\text { No serious } \\
\text { inconsistency }\end{array}$ & $\begin{array}{l}\text { No serious } \\
\text { indirectness }\end{array}$ & $\begin{array}{l}\text { No serious } \\
\text { imprecision }\end{array}$ & None & 154 & 144 & - & $\begin{array}{c}\text { not } \\
\text { pooled }\end{array}$ & $\begin{array}{l}\oplus \oplus \oplus \mathrm{O} \\
\text { Moderate }\end{array}$ & Important \\
\hline
\end{tabular}

1 All included studies conducted open label trials, which would lead to performance bias.

2 Publication bias was detected by funnel plot.

3 Considerable heterogeneity was detected between studies, which was not explained by study design, interventions, or length of follow-up.

FIgURE 3: Evidence quality for each outcome.

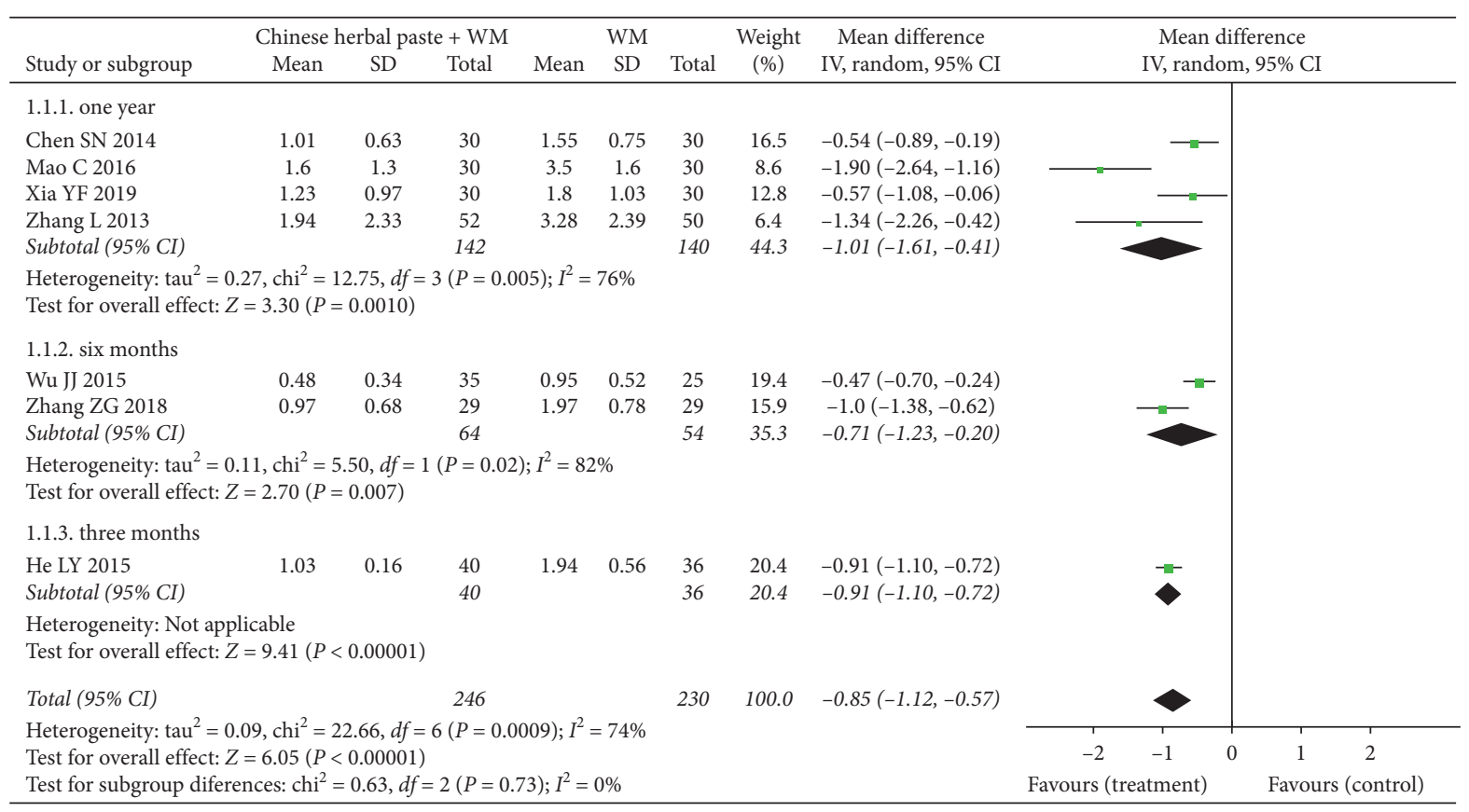

FIGURE 4: Forest plot of exacerbation frequency.

3.4.2. SGRQ Scores. SGRQ scores were evaluated in five studies $[20,21,24,34,41]$.

After the meta-analysis of the fixed-effect model, one trial [34] with treatment continued six months showed a better effect favoring Chinese herbal paste plus WM compared with WM alone (MD: $-4.50,95 \% \mathrm{CI}:-5.40$ to $-3.60 ; P<0.00001$ ) (Figure 5); four studies $[20,21,24,41]$ with two to three months' treatment duration confirmed that the combination of herbal paste and WM significantly decreased the SGRQ scores compared with WM alone (MD: -7.03 , 95\% CI: -8.69 to $-5.37 ; P<0.00001 ; I^{2}=0 \%$ ) (Figure 5 ). No statistical heterogeneity was detected. More importantly, the magnitude of these changes of SGRQ scores was greater than the minimal clinically important difference (MCID) ( -4.0 points) [43]. 


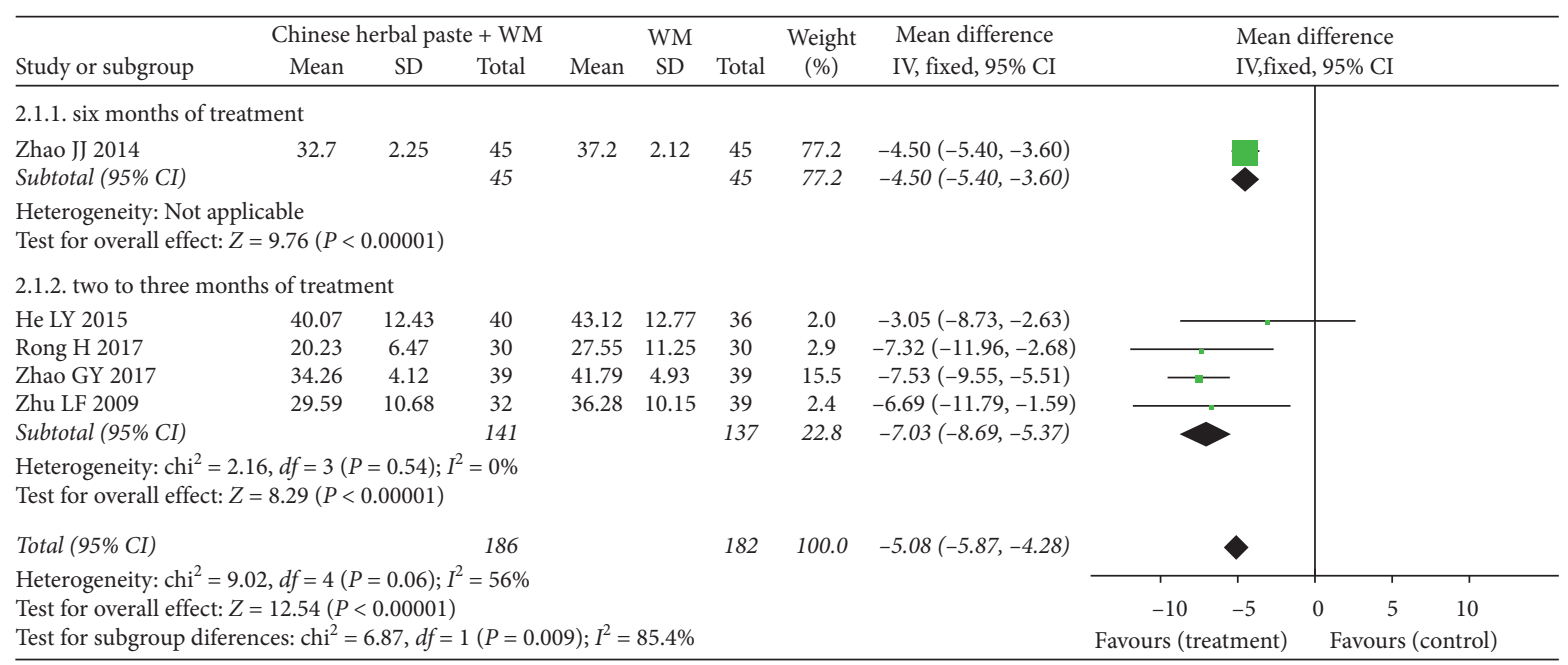

FIGURE 5: Forest plot of SGRQ scores.

3.4.3. CAT Scores. Nine studies examined CAT scores $[20,23,30,32,35,36,39,41,42]$, and the pooled analysis was performed by using a random-effects model. The subgroup analysis showed that the combination of herbal paste plus WM was superior to WM alone in terms of CAT scores in one RCT [35] after six months of treatment (MD: $-1.73,95 \%$ CI: -3.34 to $-0.12 ; P=0.04$ ) (Figure 6 ). However, the magnitude of this reduction was less than the MCID of CAT (-2 points) [44]. Eight studies [20, 23, 30, 32, 36, 39, 41, 42] with two to three months of treatment showed that CAT scores of patients in the group of herbal paste therapy combined with WM were significantly lower than those in the control group (MD: $-3.94,95 \% \mathrm{CI}:-5.99$ to -1.89 ; $P=0.0002 ; I^{2}=89 \%$ ) (Figure 6 ), and the reduction in CAT scores was greater than the MCID [44]. After examining the forest plot, we found that the study by only Zhang ZG 2018 [42] had little overlap with the summary estimate of the subgroup, and our sensitivity analysis found that exclusion of this study led to a significant reduction of heterogeneity (MD: -2.72 , 95\% CI:-3.52 to $-1.93 ; P<0.001 ; I^{2}=20 \%$ ). However, after examining the characteristics of the patients and study design of the included studies in Table 2 and Table 3, we were unable to identify the factors that might lead to the change of heterogeneity.

3.4.4. Clinical Effective Rates. Eleven trials $[21,24,29-31,33,34,37,38,41,42]$ reported the total clinical effective rates between Chinese oral herbal paste combined with WM and control groups using WM alone. The results of the subgroup meta-analysis from our fixed-effects model demonstrated that the combined use of herbal paste and WM had a better effect when compared with WM alone after six months (RR: 1.21, 95\% CI: 1.09 to $1.34 ; P=0.0002$; $I^{2}=0 \%$ ) (Figure 7 ) $[33,34,38]$, or two to three months of treatment (RR: 1.26, 95\% CI: 1.16 to $1.37 ; P<0.00001$; $I^{2}=0 \%$ ) (Figure 7) [21, 24, 29-31, 37, 41, 42]. However, the visual asymmetric funnel plot of the 11 studies showed potential publication bias (Figure 8).
3.4.5. Scores of TCM Syndrome. A total of eight trials reported scores of TCM syndrome [24, 29, 30, 32-34, 37, 42]. Subgroup analysis from the random-effects model showed that the syndrome scores of the treatment group was lower than that of the control group after six months of treatment (MD: $-4.47,95 \%$ CI: -9.66 to $0.72 ; P=0.090 ; I^{2}=96 \%$ ) (Figure 9) $[33,34]$. However, the result was not statistically significant. Another subgroup analysis with six trials $[24,29,30,32,37,42]$ showed that the combined treatment group had greater reduction in TCM syndrome scores than WM after two to three months of intervention (MD: -3.20 , 95\% CI: -5.62 to $-0.78 ; P=0.010 ; I^{2}=89 \%$ ) (Figure 9).

3.4.6. Pulmonary Function. Lung function represented by $\mathrm{FEV}_{1}$ \%pred was reported in twelve studies $[21-24,29,30,32,35-37,40,42]$. Due to the considerable heterogeneity, we tried to conduct meta-analysis by randomeffects model. In the subgroup analysis by treatment duration, one study [35] showed no significant statistical difference in $\mathrm{FEV}_{1}$ \%pred between treatment group and control group after six months of treatment (MD: 0.14, 95\% CI: -5.32 to $5.60 ; P=0.960$ ) (Figure 10 ). However, pooled analysis of the other eleven studies $[21-24,29,30,32,36,37,40,42]$ with treatment of two to three months showed that the improvement of $\mathrm{FEV}_{1} \%$ pred in the treatment group was superior to the improvement of WM alone (MD: 3.09, 95\% CI: 1.49 to 4.69; $P=0.0002$; $\left.I^{2}=66 \%\right)$ (Figure 10).

3.4.7. 6WMD. A total of five RCTs $[30,36,37,40,42]$ reported $6 \mathrm{MWD}$, all of which had a treatment duration of two to three months. Compared with WM alone, Chinese herbal paste plus WM had a better improvement on 6MWD after meta-analysis by random-effects model (MD: 48.96, 95\% CI: 22.55 to $72.37 ; P<0.001 ; I^{2}=81 \%$ ) (Figure 11 ). And the improvement in 6MWD was higher than in the MCID (30 meters) [45]. 


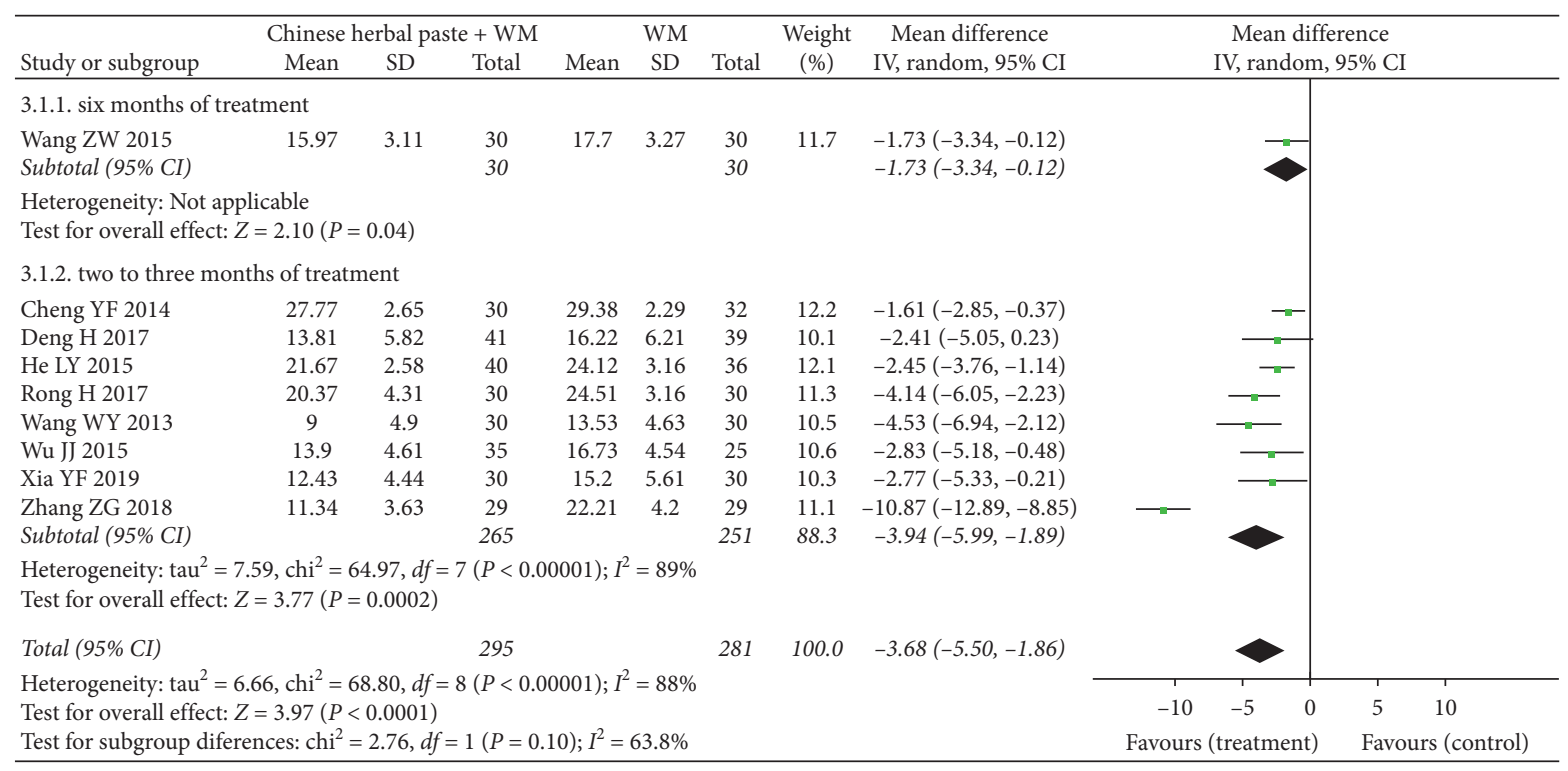

FIGURE 6: Forest plot of CAT scores.

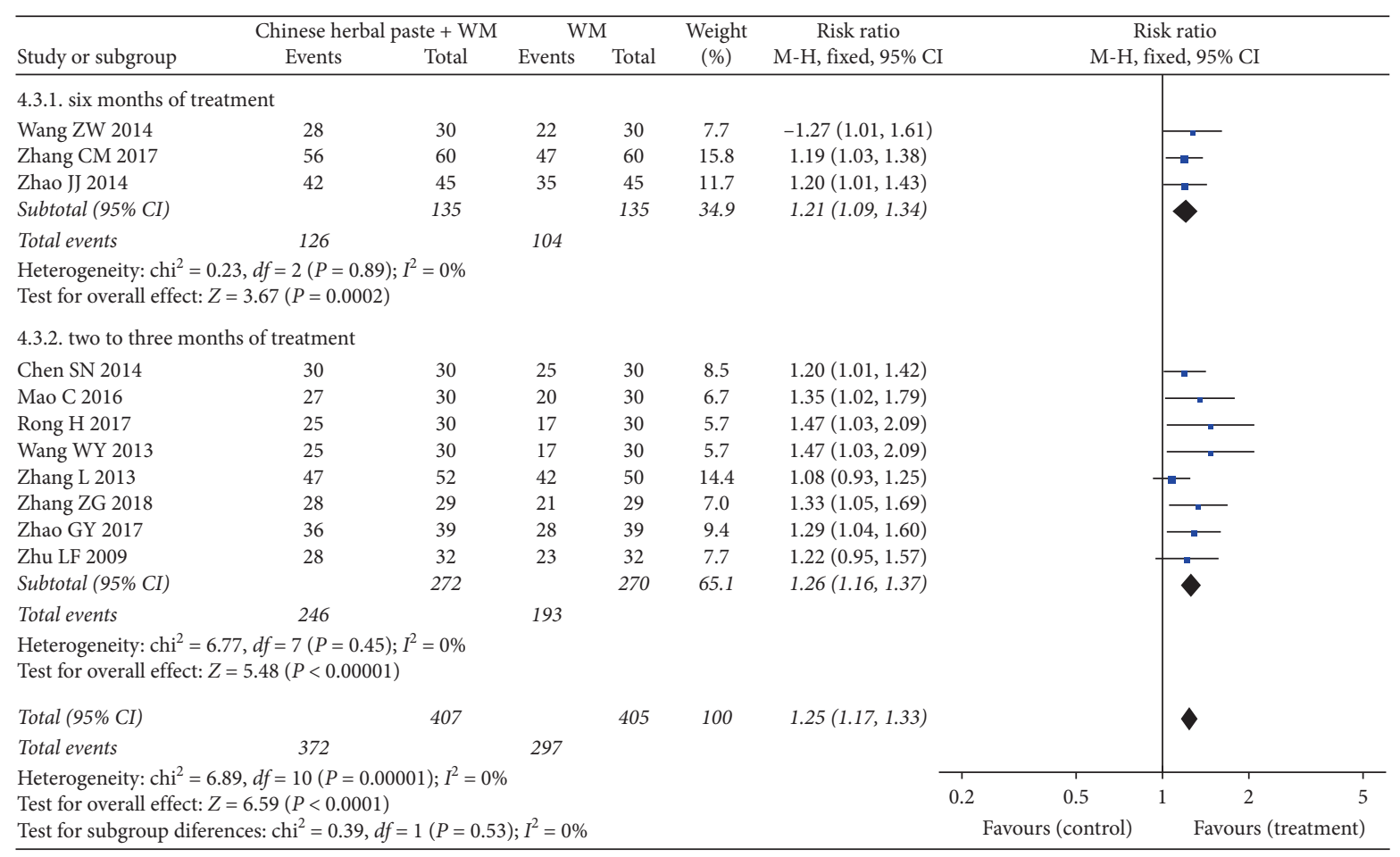

Figure 7: Forest plot of clinical effective rates.

3.4.8. Adverse Events. Six studies [22, 24, 30, 40-42] reported adverse reactions, of which none of the three studies $[30,40,41]$ had any adverse events during the trial period. Mild adverse reactions including vomiting, loose stool, abdominal distension, or sore pharynx were reported in the treatment groups of three other studies [22, 24, 42]. However, the three RCTs [22, 24, 42] showed that all adverse reactions were resolved spontaneously after long-term adherence to medication and no serious adverse events occurred (Table 4).

\section{Discussion}

Due to persistent airway inflammation and airflow limitation, patients with stable COPD have been continuingly suffering from clinical symptoms and exacerbations despite receiving standard treatments recommended by the current guidelines, which results in low quality of life and high mortality [46]. Numerous studies have showed that the incidence of COPD exacerbation in winter is obviously increasing, which may be driven by increased pathogen as 


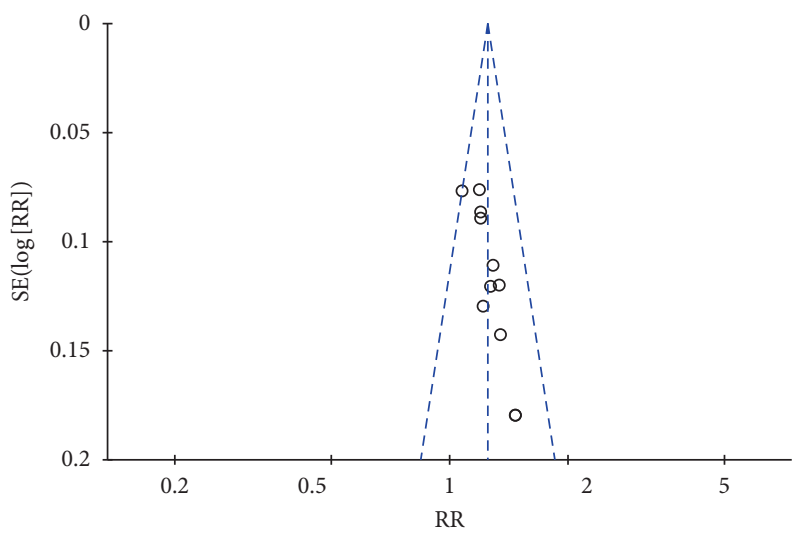

FIGURE 8: Funnel plot of clinical effective rates.

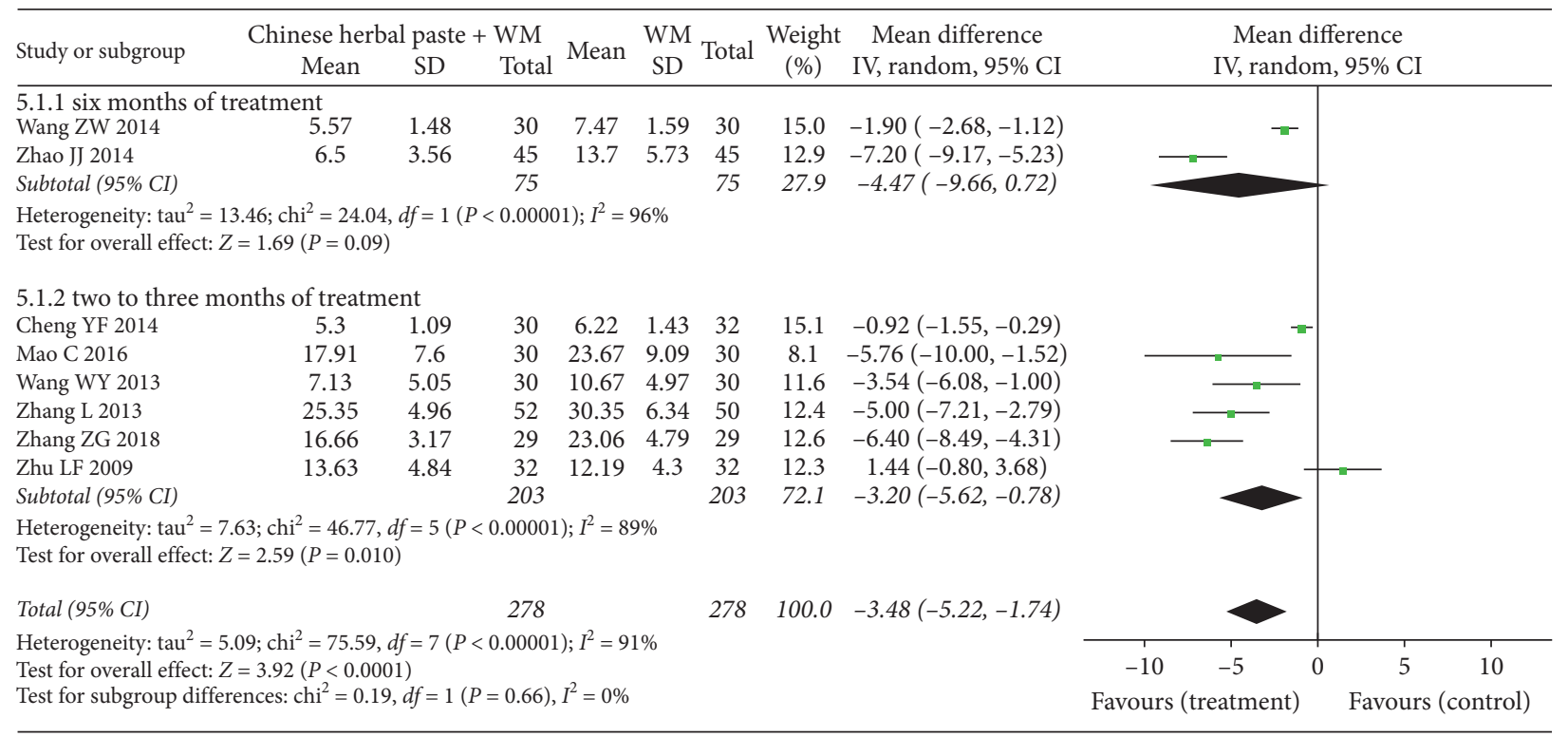

Figure 9: Forest plot of scores of TCM syndrome.

well as the interaction between low temperatures and poor immunity [47-49]. The prescription of Chinese herbal paste is individualized based on the theory of "treatment based on syndrome differentiation", with the main purpose of replenishing deficiency, resisting external evil to strengthen immunity against infection of various pathogens [17]. The current systematic review of 19 RCTs with 1303 individuals showed that Chinese herbal paste has a good adjuvant therapeutic effect on stable COPD. The results of pooled analysis demonstrated that oral herbal paste for two to three months in the winter may help to prevent the risk of future acute exacerbations, improve quality of life, clinical symptoms, and lung function, and increase activity endurance.

Inhaler therapies with bronchodilator and/or glucocorticoids are the most important part of managing stable COPD. However, low inhalation flow resulting from poor lung function, aging, cognitive impairment, and presence of comorbidities may affect correct use of inhaler device, which led to patients' reduced adherence with inhaler therapy [50]. Additionally, inhaler misuse was associated with increased risk of exacerbations, courses of oral steroids, control of clinical symptoms, and overall health care costs [51]. Therefore, a large number of patients with COPD tried to improve their health status through traditional medical therapies, and CHM was one of them. Chinese herbal paste is a type of TCM treatment that is different from CHM decoction and modern inhalant drugs, which gains better patient adherence due to its satisfying taste, several milliliters' dosage each time, and no need for long-term use.

In several subgroup analyses, we found that the combined treatment of Chinese herbal paste and WM for six months had an advantage over WM alone in SGRQ score, CAT score, and clinical effective rate. However, the benefits in lung function and score of TCM syndrome were not statistically significant. This may due to fewer original studies with treatment duration of 6 months. Chinese herbal paste is usually recommended for use in during winter for 2 to 3 months, and our study results also demonstrated the treatment effects of 2 to 3 months. All included studies compared the differences of quality of life, clinical 


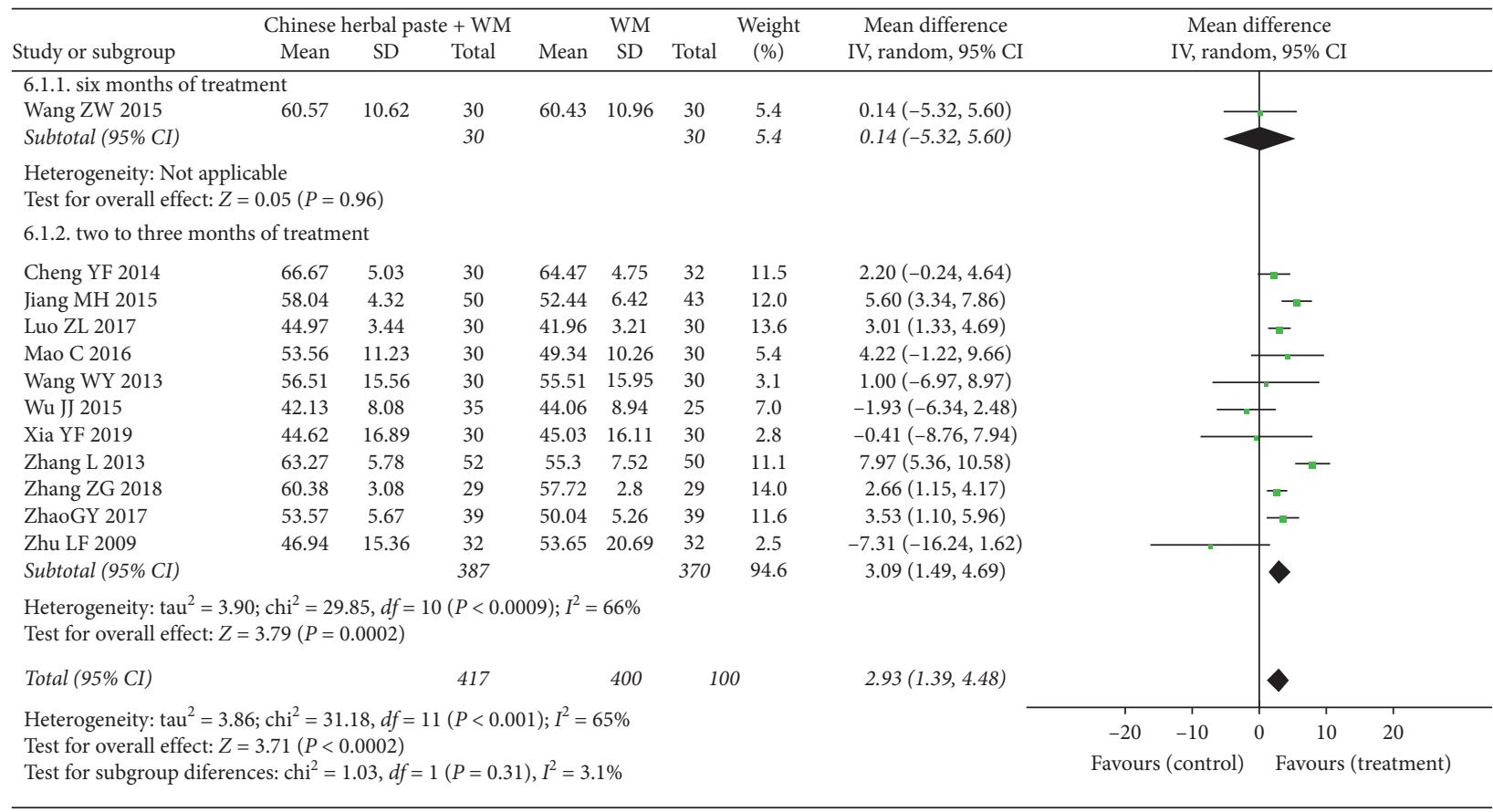

Figure 10: Forest plot of $\mathrm{FEV}_{1} \%$ pred.

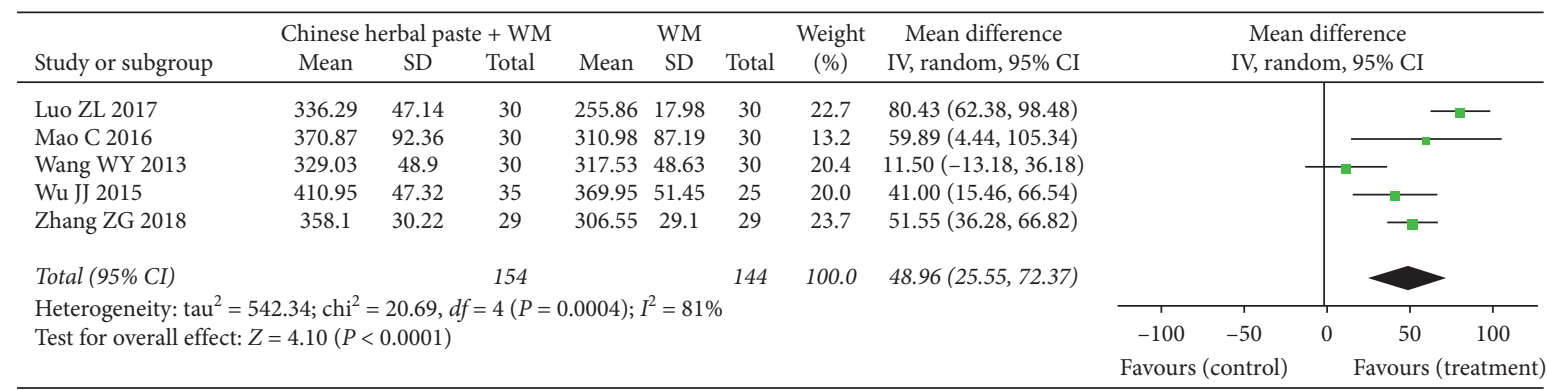

FIgURE 11: Forest plot of 6MWD.

TABLE 4: Adverse events of included RCTs.

\begin{tabular}{|c|c|c|c|c|c|c|c|c|c|}
\hline $\begin{array}{l}\text { Author, } \\
\text { year }\end{array}$ & $\begin{array}{l}\text { Size } \\
(\mathrm{T} / \mathrm{C})\end{array}$ & $\begin{array}{c}\text { Total } \\
\text { number of } \\
\text { adverse } \\
\text { events } \\
\text { (T/C) }\end{array}$ & $\begin{array}{l}\text { Withdrawal } \\
\text { due to adverse } \\
\text { events }(\mathrm{T} / \mathrm{C})\end{array}$ & $\begin{array}{l}\text { Emesis } \\
(\mathrm{T} / \mathrm{C})\end{array}$ & $\begin{array}{c}\text { Loose } \\
\text { stool } \\
(\mathrm{T} / \mathrm{C})\end{array}$ & $\begin{array}{l}\text { Abdominal } \\
\text { distension } \\
(\mathrm{T} / \mathrm{C})\end{array}$ & $\begin{array}{l}\text { Gastrointestinal } \\
\text { discomfort }(\mathrm{T} / \mathrm{C})\end{array}$ & $\begin{array}{l}\text { Pharyngalgia } \\
\text { (T/C) }\end{array}$ & $\begin{array}{c}\text { Laboratory } \\
\text { test index } \\
(\mathrm{T} / \mathrm{C})\end{array}$ \\
\hline $\begin{array}{l}\text { Zhu et al. } \\
2009 \text { [24] }\end{array}$ & $32 / 32$ & $3 / 0$ & $0 / 0$ & $0 / 0$ & $0 / 0$ & $0 / 0$ & $3 / 0$ & $0 / 0$ & $0 / 0$ \\
\hline $\begin{array}{l}\text { Wang and } \\
\text { Liu } 2013 \\
{[30]}\end{array}$ & $30 / 30$ & $0 / 0$ & $0 / 0$ & $0 / 0$ & $0 / 0$ & $0 / 0$ & $0 / 0$ & $0 / 0$ & $0 / 0$ \\
\hline $\begin{array}{l}\text { Jiang et al. } \\
2015 \text { [22] }\end{array}$ & $50 / 43$ & $10 / 0$ & $0 / 0$ & $2 / 0$ & $5 / 0$ & $3 / 0$ & $0 / 0$ & $0 / 0$ & $0 / 0$ \\
\hline $\begin{array}{l}\text { Luo } 2017 \\
{[40]}\end{array}$ & $30 / 30$ & $0 / 0$ & $0 / 0$ & $0 / 0$ & $0 / 0$ & $0 / 0$ & $0 / 0$ & $0 / 0$ & $0 / 0$ \\
\hline $\begin{array}{l}\text { Rong and } \\
\text { Ding } 2017 \\
\text { [41] }\end{array}$ & $30 / 30$ & $0 / 0$ & $0 / 0$ & $0 / 0$ & $0 / 0$ & $0 / 0$ & $0 / 0$ & $0 / 0$ & $0 / 0$ \\
\hline $\begin{array}{l}\text { Zhang } \\
2018 \text { [42] }\end{array}$ & $29 / 29$ & $1 / 0$ & $0 / 0$ & $0 / 0$ & $0 / 0$ & $0 / 0$ & $0 / 0$ & $1 / 0$ & $0 / 0$ \\
\hline
\end{tabular}


symptoms, lung function, and activity endurance before and after treatment, and the long-term effects of herbal paste after drug withdrawal need to be confirmed by high-quality studies in the future.

MCID is the smallest difference of measurable clinical parameter in the absence of troublesome side effects, which represents a meaningful change for the better or worse that is perceived by the clinician and patient [52]. In this systematic review, we used MCID to confirm whether the changes of clinical outcomes were clinically meaningful. Our study showed that regardless of treatment duration of two to three months or six months, the improvement in SGRQ score was above the MCID. Meanwhile, in the treatment duration of two to three months, the CAT score and 6WMD significantly exceeded the MCID. However, after 6-month treatment, the improvement in the CAT score did not reach the MCID. Although the results of meta-analysis of the CAT score and the 6MWD indicated certain heterogeneity, the direction of effect size in the sensitivity analysis did not change, and the effect size was maintained above the MCID.

Safety analysis of included studies showed that Chinese oral herbal paste was well tolerated and had no serious adverse events in the treatment of stable COPD. However, most of the studies did not examine safety indicators in detail such as biochemical indexes of blood. Due to the numerous compositions of herbal and complex compatibility, Chinese herbal paste should be prescribed following as per patients' constitution and the degree of qi, blood, and yin or yang deficiency to achieve reasonable compatibility to avoid inappropriate or excessive supplementation [53]. Therefore, more in-depth clinical and pharmacologically basic studies are needed in the future to explore the therapeutic benefits of herbal paste.

Heterogeneity between all the included studies decreased after we performed a subgroup analysis based on the differences in treatment duration or follow-up time between studies. However, our meta-analysis showed considerable heterogeneity in terms of several outcome measurements (e.g. exacerbation frequency, CAT scores, scores of TCM syndrome, $\mathrm{FEV}_{1} \%$ pred, and 6MWD). The heterogeneity was not reduced significantly after subgroup analyses such as TCM syndrome, which may be mainly due to poor reporting quality and lack of clinical homogeneity. Most trials did not comply with Consolidated Standards of Reporting Trials (CONSORT) statement and the Herbal Interventions promotion to conduct standard clinical studies [54, 55]. Additionally, the clinical practice of TCM is characterized by syndrome differentiation and treatment, which leads to various drug ingredients, dosages, and administration frequency of herbal paste. Furthermore, due to the lack of corresponding complete data, the baseline parameters (such as severity and course of disease) of participants among some studies may also be inconsistent, which may contribute to heterogeneity in the pooling of results.

During the evaluation process, we sought to prevent and avoid any potential bias. A comprehensive search of relevant databases for published and unpublished studies was performed. However, different studies chose different outcome indicators or measurement methods, which led to few original trials of partial results and limited the ability of pooling analysis for the results. The quality of the evidence from included studies ranged from very low to moderate for a variety of reasons. Firstly, most of RCTs adequately described the details of the generated sequences, however the method of randomization was not reported in the five studies $[21,31,32,36,38]$, which raised doubts about the validity of randomization. Secondly, it is difficult to produce placebo with similar appearance, smell, and dosage form for oral herbal paste, therefore the placebo control was not well performed in these studies. In our systematic review, except for one singleblinded study [24], the other studies did not report blinding and allocation concealment. Therefore, all included trials were considered to have a high risk of bias in the blinding field. Finally, all included studies were conducted in China and the studies published in other languages than English and Chinese were not included, which may lead to publication bias.

\section{Conclusions}

Overall, this systematic review and meta-analysis showed that on the basis of WM therapy, treatment with Chinese oral herbal paste for 2 to 3 months is an effective auxiliary therapy for stable COPD, which may reduce acute exacerbation, improve quality of life, clinical symptoms, 6WMD, and lung function, and ameliorate TCM symptom scores and clinical effective rate. In addition, herbal paste has small side effects and was well tolerated. However, considering the heterogeneity and risk of bias of included studies, highquality and well-designed RCTs are needed in the future to further explore the clinical efficacy and underlying mechanisms of Chinese oral herbal paste.

\section{Abbreviations \\ COPD: Chronic obstructive pulmonary disease \\ CHM: Chinese herbal medicine \\ CAT: COPD assessment test \\ CI: $\quad$ Confidence interval \\ FEV1\% Forced expiratory volume in one second as a \\ pre: percentage of the predicted value \\ GRADE: Grading of recommendations assessment, development, and evaluation \\ MCID: Minimal clinically important difference \\ MD: $\quad$ Mean difference \\ 6MWD: 6-minute walk distance \\ PSSR: Percentage of the symptom score reduction \\ RCTs: $\quad$ Randomized controlled trials \\ RR: $\quad$ Relative risk \\ SGRQ: St George's respiratory questionnaire \\ TCM: Traditional Chinese medicine \\ WM: Western medicine.}

\section{Conflicts of Interest}

All authors have no conflicts of interest to declare. 


\section{Authors' Contributions}

Yan Zeng, Juan-juan $\mathrm{Fu}$, and Bing Mao conceived and designed this study. Yan Zeng and $\mathrm{Yu} \mathrm{Li}$ drafted the manuscript. Yan Zeng, Chan Xiong, Yu Li, Li Liao, and Hua Wei assisted in the study selection, data extraction, and quality assessment. Statistical analysis was performed by Yan Zeng and Ti-wei Miao. Juan-juan Fu and Bing Mao revised the manuscript. All authors read and approved the final manuscript.

\section{Acknowledgments}

The authors thank the Cochrane Collaboration Group for providing Rev Man 5.3 software for statistical analysis and construction of forest plot. This study is supported by the National Natural Science Foundation of China (nos. 81870014 and 81400030) and the Thousand Talents Program, Sichuan Province, China (no. 0082204151045). The funders do not participate in the design of the study and drafting the manuscript, nor take part in the collection, analysis, and interpretation of the data, and any decision to publish the results.

\section{References}

[1] M. Naghavi, A. A. Abajobir, C. Abbafati et al., "Global, regional, and national age-sex specific mortality for 264 causes of death, 1980-2016: a systematic analysis for the Global Burden of Disease Study 2016," The Lancet, vol. 390, pp. 1151-1210, 2017.

[2] Global Initiative for Chronic Obstructive Lung Disease (GOLD), Global Strategy for the Diagnosis, Management and Prevention of COPD, Global Initiative for Chronic Obstructive Lung Disease (GOLD), Madison, WI, ISA, 2019, http://www. goldcopd.com.

[3] C. Wang, J. Xu, L. Yang et al., "Prevalence and risk factors of chronic obstructive pulmonary disease in China (the China pulmonary health $\mathrm{CPH}$ study): a national cross-sectional study," The Lancet, vol. 391, no. 10131, pp. 1706-1717, 2018.

[4] T. A. R. Seemungal, G. C. Donaldson, E. A. Paul, J. C. Bestall, D. J. Jeffries, and J. A. Wedzicha, "Effect of exacerbation on quality of life in patients with chronic obstructive pulmonary disease," American Journal of Respiratory and Critical Care Medicine, vol. 157, no. 5, pp. 1418-1422, 1998.

[5] H. Qureshi, A. Sharafkhaneh, and N. A. Hanania, "Chronic obstructive pulmonary disease exacerbations: latest evidence and clinical implications," Therapeutic Advances in Chronic Disease, vol. 5, no. 5, pp. 212-227, 2014.

[6] M. Miravitlles, H. Worth, C. Juan José Soler et al., "Observational study to characterise 24-hour COPD symptoms and their relationship with patient-reported outcomes: results from the ASSESS study," Respiratory Research, vol. 15, no. 1, pp. 1-13, 2014.

[7] J. Patel, A. Coutinho, O. Lunacsek, and A. Dalal, "COPD affects worker productivity and health care costs," International Journal of Chronic Obstructive Pulmonary Disease, vol. 13, pp. 2301-2311, 2018.

[8] U. Maqsood, T. N. Ho, D. J. W. Evans et al., "Once daily longacting beta2-agonists and long-acting muscarinic antagonists in a combined inhaler versus placebo for chronic obstructive pulmonary disease," Cochrane Database of Systematic Reviews, vol. 3, 2018.

[9] M. X. Rojas-Reyes, O. M. Garcia Morales, and R. J. Dennis, "Combination inhaled steroid and long-acting beta(2)-agonist in addition to tiotropium versus tiotropium or combination alone for chronic obstructive pulmonary disease," Cochrane Database of Systematic Reviews, vol. 6, 2010.

[10] H. Ni, S. Moe, Z. Soe, K. T. Myint, and K. N. Viswanathan, "Combined aclidinium bromide and long-acting beta2-agonist for chronic obstructive pulmonary disease (COPD)," Cochrane Database of Systematic Reviews, vol. 12, 2018.

[11] D. D. Sin, T. Stafinski, Y. C. Ng, N. R. Bell, and P. Jacobs, “The impact of chronic obstructive pulmonary disease on work loss in the United States," American Journal of Respiratory and Critical Care Medicine, vol. 165, no. 5, pp. 704-707, 2002.

[12] B. Zhu, Y. Wang, J. Ming, W. Chen, and L. Zhang, "Disease burden of COPD in China: a systematic review," International Journal of Chronic Obstructive Pulmonary Disease, vol. 13, pp. 1353-1364, 2018.

[13] G. N. Rootmensen, A. R. J. van Keimpema, H. M. Jansen, and R. J. de Haan, "Predictors of incorrect inhalation technique in patients with asthma or COPD a study using a validated videotaped scoring method," Journal of Aerosol Medicine and Pulmonary Drug Delivery, vol. 23, no. 5, pp. 323-328, 2010.

[14] Chinese Society of Traditional Chinese Medicine Internal Medicine Branch Pulmonary Diseases Professional Committee, "Guidelines for traditional Chinese medicine diagnosis and treatment of chronic obstructive pulmonary disease (2011)," Journal of Traditional Chinese Medicine, vol. 53, no. 1, pp. 80-84, 2012.

[15] Chinese Pharmacopoeia Committee, Chinese Pharmacopoeia (The Fourth Part): China Medical Science and Technology, Chinese Pharmacopoeia Committee, Beijing, China, 2010.

[16] Y. Zeng, Y. Li, H. Wei et al., "Chinese oral herbal paste for the treatment of stable chronic obstructive pulmonary disease: protocol for a systematic review and meta-analysis," Medicine (Baltimore), vol. 98, no. 28, Article ID e16444, 2019.

[17] C. H. Zeng, W. C. Wang, and X. Zhang, "An analysis of the characteristics of paste in "compendium of materia medica"," Lishizhen Medicine and Materia Medica Research, vol. 29, no. 8, pp. 2009-2010, 2018.

[18] X. E. Lv and X. J. Chen, "Advances in origin and clinical application of TCM creama formula," Journal of Liaoning University of Traditional Chinese Medicine, vol. 15, no. 10, pp. 213-215, 2013.

[19] H. Y. Li, "Discussion on the preparation technology of Chinese herbal paste," Henan Traditional Chinese Medicine, vol. 33, no. 11, pp. 1930-1932, 2013.

[20] L. Y. He, M. Zang, and X. H. Chen, "Effects of winter paste on life quality and short- term prognosis of patients with chronic obstructive pulmonary disease," Journal of Shandong University of Traditional Chinese Medicine, vol. 39, no. 6, pp. 533-535, 2015.

[21] G. Y. Zhao and L. P. Li, "Effect of Bufei Huatan cream on life quality and lung function of chronic obstructive pulmonary disease in stable phase," Acta Chinese Medicine, vol. 32, no. 230, pp. 1165-1168, 2017.

[22] M. H. Jiang, Y. H. Wang, and Y. Lianfei, "Clinical analysis of Yishen Lianfei paste on the treatment of chronic obstructive pulmonary disease," Journal of Practical Traditional Chinese Medicine, vol. 31, no. 2, 2015.

[23] Y. F. Xia and Z. K. Sun, "Clinical observation on gushen bupi cream combined with seretide for the treatment of middle-old age patients with chronic obstructive pulmonary disease at 
stable phase," Journal of Liaoning University of Traditional Chinese Medicine, vol. 21, no. 5, pp. 123-126, 2019.

[24] L. F. Zhu, Y. J. Bian, and F. Zhen, "Clinical study on the effects of bufei yishen treatment on quality of life of patients with chronic obstructive pulmonary disease in stable period," Chinese Journal of Information on Traditional Chinese Medicine, vol. 16, no. 12, pp. 13-15, 2009.

[25] J. P. Higgins and S. Green, Cochrane Handbook for Systematic Reviews of Interventions, Wiley, Hoboken, NJ, USA, 2018.

[26] Chinese Medical Association Respiratory Diseases Society Chronic Obstructive Pulmonary Diseases Group, "Guidelines for the diagnosis and treatment of chronic obstructive pulmonary disease (revised 2013)," Chinese Journal of the Frontiers of Medical Science, vol. 6, no. 3, pp. 67-80, 2014.

[27] X. Y. Zhen, Guiding Principle of Clinical Research on New Drugs of Traditional Chinese Medicine (Trial Implementation), Chinese Medical Science and Technology Press, Beijing, China, 2002.

[28] G. H. Guyatt, A. D. Oxman, G. E. Vist et al., "GRADE: an emerging consensus on rating quality of evidence and strength of recommendations," BMJ, vol. 336, no. 7650, pp. 924-926, 2008.

[29] L. Zhang, H. Zhao, and H. Ji, "Clinical observation on paste for chronic obstructive pulmonary disease (lung and kidney deficiency) at stable phase," Journal of Sichuan of Traditional Chinese Medicine, vol. 31, no. 6, pp. 106-107, 2013.

[30] W. Y. Wang and J. B. Liu, "Clinical observation of Feikang paste in the treatment of chronic obstructive pulmonary disease at stable stage (lung and spleen deficiency syndrome)," Journal of New Chinese Medicine, vol. 45, no. 11, pp. 27-29, 2013.

[31] S. N. Chen, R. X. Li, and Y. Feng, "Observation of therapeutic effect of Shuyuhuo paste for chronic obstructive pulmonary disease," Journal of Guangxi University of Chinese Medicine, vol. 17, no. 2, pp. 22-24, 2014.

[32] Y. F. Cheng, K. J. Ma, and R. He, "Clinical observation on Bushen Gujin paste for the treatment of chronic obstructive pulmonary disease," Clinical Journal of Traditional Chinese Medicine, vol. 26, no. 8, pp. 798-800, 2014.

[33] Z. W. Wang, P. L. Yang, and L. Shen, "Clinical efficacy and mechanism of Baibu Yangfei decoction for chronic obstructive pulmonary disease," Jilin Journal of Chinese Medicine, vol. 34, no. 9, pp. 899-902, 2014.

[34] J. J. Zhao, J. P. Qing, and S. F. Tang, "Clinical research of Bufei Huazhuo cream party treatment on chronic obstructive pulmonary disease at stable phase," Chinese Journal of Experimental Traditional Medical Formulae, vol. 20, no. 7, pp. 216-219, 2014.

[35] Z. W. Wang, P. L. Yang, and L. Shen, "Clinical study ofBaiBu YangFei decocted extract in the treatment for COPD patients at stable stage," Western Journal of Traditional Chinese Medicine, vol. 28, no. 5, pp. 56-59, 2015.

[36] J. J. Wu, H. R. He, and G. Sun, "The clinical research of applying paste as treatment for patients in COPD stabilaization period," China Journal of Chinese Materia Medica, pp. 1823-1824, 2015.

[37] C. Mao, "Clinical study of pingchuan guben paste in the treatment of patients with chronic obstructive pulmonary diseases," Journal of Liaoning University of Traditional Chinese Medicine, vol. 18, no. 6, pp. 170-172, 2016.

[38] C. M. Zhang, J. Q. Mao, and Y. C. Chen, "Clinical study of Bufei Zhike ointment combined with seretide in the treatment of stable chronic obstructive pulmonary disease," Acta Chinese Medicine, vol. 32, no. 235, pp. 2323-2325, 2017.
[39] H. Deng, X. G. Zhang, and L. Wang, "Clinical study on Jianpi Lifei paste in the treatment of chronic obstructive pulmonary disease at stable stage," Journal of Shaanxi University of Chinese Medicine, vol. 40, no. 2, pp. 34-36, 2017.

[40] Z. L. Luo, The Effect of Fufei Gushen Paste on BODE Index during Stable Phase in Patients with Chronic Obstructive Pulmonary Disease, Guangxi University of Traditional Chinese Medicine, Nanning, China, 2017.

[41] H. Rong and N. Ding, "Clinical research on Zijin Bushui paste in treatment of chronic obstructive pulmonary disease in elderly adult," Journal of Hubei University of Chinese Medicine, vol. 19, no. 2, pp. 23-26, 2017.

[42] Z. G. Zhang, Clinical Curative Observation on Bufei Yishen Ointment for the Treatment of Chronic Obstructive Pulmonary Disease(Deficiency of Lung and Kidney) on Stable Phase, Hunan University of Traditional Chinese Medicine, Changsha, China, 2018.

[43] F. J. Martinez, J. F. Donohue, and S. I. Rennard, "The future of chronic obstructive pulmonary disease treatment-difficulties of and barriers to drug development," The Lancet, vol. 378, no. 9795, pp. 1027-1037, 2011.

[44] S. S. C. Kon, J. L. Canavan, S. E. Jones et al., "Minimum clinically important difference for the COPD assessment test: a prospective analysis," The Lancet Respiratory Medicine, vol. 2, no. 3, pp. 195-203, 2014.

[45] M. I. Polkey, M. A. Spruit, W. Wouters, L. D. Edwards, R. TalSinger, and C. Celli, "Six-minute-walk test in chronic obstructive pulmonary disease minimal clinically important difference for death or hospitalization," American Journal of Respiratory and Critical Care Medicine, vol. 187, no. 4, pp. 382-386, 2013.

[46] M. K. Han, C. H. Martinez, D. H. Au et al., "Meeting the challenge of COPD care delivery in the USA: a multiprovider perspective," The Lancet Respiratory Medicine, vol. 4, no. 6, pp. 473-526, 2016.

[47] C. R. Jenkins, B. Celli, J. A. Anderson et al., "Seasonality and determinants of moderate and severe COPD exacerbations in the TORCH study," European Respiratory Journal, vol. 39, no. 1, pp. 38-45, 2012.

[48] T. M. A. Wilkinson, E. Aris, S. Bourne et al., "A prospective, observational cohort study of the seasonal dynamics of airway pathogens in the aetiology of exacerbations in COPD," Thorax, vol. 72, no. 10, pp. 919-927, 2017.

[49] J. Miguel-Diez, J. Hernandez-Vazquez, A. Lopez-de-Andres, A. Álvaro-Meca, V. Hernández-Barrera, and R. JiménezGarcía, "Analysis of environmental risk factors for chronic obstructive pulmonary disease exacerbation: a case-crossover study (2004-2013)," PLoS One, vol. 14, no. 5, Article ID e0217143, 2019.

[50] I. Sulaiman, B. Cushen, G. Greene et al., "Objective assessment of adherence to inhalers by patients with chronic obstructive pulmonary disease," American Journal of Respiratory and Critical Care Medicine, vol. 195, no. 10, pp. 1333-1343, 2017.

[51] A. S. Melani, M. Bonavia, V. Cilenti et al., "Inhaler mishandling remains common in real life and is associated with reduced disease control," Respiratory Medicine, vol. 105, no. 6, pp. 930-938, 2011.

[52] B. Make, "How can we assess outcomes of clinical trials: the MCID approach," COPD: Journal of Chronic Obstructive Pulmonary Disease, vol. 4, no. 3, pp. 191-194, 2009.

[53] D. D. Chen, "Analysis of common adverse reactions of Chinese herbal paste," Henan Traditional Chinese Medicine, vol. 35, no. 3, pp. 510-511, 2015. 
[54] J. Gagnier, H. Boon, P. Rochon, J. Barnes, D. Moher, and C. Bombardier, "Improving the quality of reporting of randomized controlled trials evaluating herbal interventions: implementing the CONSORT statement corrected," Explore, vol. 2, no. 2, pp. 143-149, 2006.

[55] K. F. Schulz, D. G. Altman, and D. Moher, "CONSORT 2010 statement: updated guidelines for reporting parallel group randomized trials," Obstetrics \& Gynecology, vol. 115, no. 5, pp. 1063-1070, 2010. 